\title{
Communication- and position-aware reconfigurable route optimization in large-scale mobile sensor networks
}

\author{
Adnan Fida*, Muhammad lqbal and Trung Dung Ngo
}

\begin{abstract}
We consider the problem of route selection and optimization for a mobile sensor network, which involves two nodes transferring information over a large area using a number of intermediate routers in the presence of noise, path loss, multipath fading, and interference. The communication- and position-aware reconfigurable (COMPARE) route optimization framework is proposed to improve the end-to-end throughput of the routes in the realistic communication environment. Initially, a communication-aware route selection strategy selects the optimal route by integrating the routing decisions with the link quality. The quality of the wireless link is characterized by the reception probability, i.e., the probability of successfully receiving packets over a realistic communication link. The selected route is reconfigured by exploiting the multipath fading, position information, and the mobility of the nodes during the phase of position-aware optimization. The optimal position for a router is heuristically found using a priori information about fading channels and the positions of transmitting and interfering nodes. The router is guided to the optimal position using the feedback mobility control, and only the route selected by the COMPARE framework is used for data transmission between the source and destination node. We illustrate through simulations that the proposed framework provides routes with considerably better performance than conventional route selection metrics, in terms of the end-to-end throughput.
\end{abstract}

Keywords: Mobile sensor networks; Realistic communication model; Position-aware optimization; Particle swarm optimization

\section{Introduction}

Mobile sensor networks (MSNs) could play an important role in future applications such as rescue operations, target tracking, and environmental scanning. The guiding vision behind mobile sensor network research is that mobile platforms (nodes) embedded with sensors can navigate and visit targets in a two-dimensional environment in order to achieve a common goal. The nodes in such networks need communication links that offer a high quality of service (QoS), allowing them to deliver large bandwidth information in forms such as continuous images or real-time videos. Achieving a high QoS in MSNs poses several challenges due to uncertainties in the methods of communication, routing, and sensing. The transmitted

*Correspondence: adnan fida@hotmail.com

The More Than One Robotics Laboratory, Faculty of Science, University Brunei Darussalam, Tungku Link Rd, BE1410, Bandar Seri Begawan, Brunei waves are attenuated, reflected, and refracted from objects in the environment, resulting in degradation of the performance of the communication links. Since the nodes need to spread out geographically, direct transmission may not be feasible and ad hoc routing mechanisms may need to be deployed to relay the data packets between nodes [1]. This necessitates multihop routing where nodes also act as routers forwarding data to other nodes as shown in Figure 1. The first problem is to select the optimal route out of many available routes. In single-path routing, only the selected route out of many available routes is then used for data transmission. Once the route is established, the fundamental challenge is to guarantee a robust flow of information [2-4]. The flow of information can be optimized via route reconfiguration, which involves the routers positioning themselves in a manner that ensures robust communication between the source and destination nodes.

\section{Springer}

(c) 2014 Fida et al: licensee Springer. This is an Open Access article distributed under the terms of the Creative Commons Attribution License (http://creativecommons.org/licenses/by/4.0), which permits unrestricted use, distribution, and reproduction in any medium, provided the original work is properly credited. 


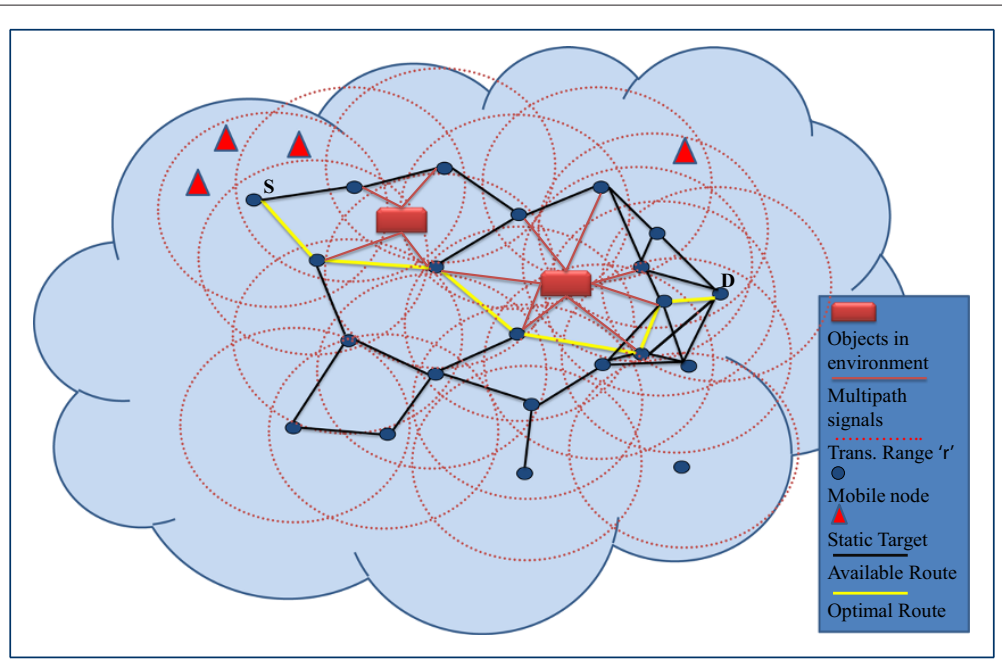

Figure $1 \mathrm{~A}$ mobile sensor network where nodes are deployed to find the static target/victim in a particular region. The node can directly communicate with other nodes inside the transmission range $r$. The communication with nodes outside the transmission range is achieved via multihop communication. The signal arrives at the receiver node via a number of paths. The source node $S$ requires optimal route out of many available routes to send data to the sink node $D$ via intermediate routers.

\subsection{Related work}

\subsubsection{Communication-aware route selection}

Due to the special demands of mobility, the routing protocols for traditional wireless sensor networks are not suitable for MSNs and existing literature available for mobile ad hoc networks may be inherited. The performance analysis of conventional route establishment schemes in ad hoc networks is based on over-simplified communication link models such as the binary link model where nodes can perfectly communicate within a transmission radius and nothing at all is communicated outside that radius [5]. Other approaches consider free space models where signal strength decays according to path loss and successful transmission is possible within a deterministic transmission radius irrespective of wireless link conditions [6-8]. The simplistic assumptions used in such models yield well-documented limitations and unrealistic conclusions [9]. The routes for data communication are determined on the basis of route selection metrics. However, route selection metrics based on the simplified communication models do not generate reliable routes in realistic scenarios as they neglect the sensitivity of the wireless links to noise and fading. For instance, the hop count (HC) metric, which is the most widely used route selection metric in $\mathrm{ad}$ hoc networks $[10,11]$, is based on the notion of the ideal communication link model, i.e., either communication is perfect or no communication at all. The use of geographical position is another popular approach for route selection in ad hoc networks [12]. From the perspective of wireless link quality, the usage of Euclidean distance (ED) as metric for route selection is based on free space models so that it only considers the path loss due to distance.
Consequently, $\mathrm{HC}$ selects suboptimal routes as it prefers long links irrespective of their quality to minimize hop count [13] and ED-based routes overlook the fact that link quality can be significantly decreased over small distances due to the multipath fading effects.

Link quality is increasingly being taken into account for routing decisions in wireless sensor networks. Chen et al. in [14] presented a dynamic window concept to record the link information and proposed link quality estimationbased routing protocol (LQER). Sequential assignment routing (SAR) [15] developed by Sohrabi et al. incorporates some notion of QoS in its routing decisions. The work in [15] was extended by Akkaya and Younis in [16], where their proposed scheme selects routes that meet the end-to-end delay requirements and provide maximum throughput. However, these works did not consider the drastic effects of path loss, fading, and interference on the link quality. An awareness of the properties of communication channels and the possibility of interference must be taken into account to provide a high QoS in a realistic environment. In [17], we developed a communicationaware route selection strategy for static nodes which incorporated the inherent uncertainty in the performance of realistic wireless communication links due to noise, fading, and accumulated interference among the nodes.

\subsubsection{Position-aware optimization}

We aim to find an optimal position or continuum of positions with better communication quality. The problem of optimum positioning of routers has received significant attention in the robotics and sensor community. To solve this problem, the algebraic-graph approaches are 
proposed by Kim and Mesbahi [18] and Gennaro and Jadbabaie [3]. In [18], an iterative greedy-type algorithm is proposed to maximize the Fiedler eigenvalue for finding optimal configuration of wireless sensor nodes. Gennaro and Jadbabaie [3] use a sub-gradient algorithm for computing the second eigenvector to maximize the Fiedler eigenvalue. Although the Fiedler eigenvalue is a good measure of graph connectivity in undirected networks, it does not reflect the reception quality of communication. The communication-oriented metric, i.e., the capacity, has been used by Dixon and Frew [19], but it does not measure the current communication quality. Xu et al. [20] and Wang et al. [21] investigated the optimal positioning of relay nodes in relation to network lifetime and connectivity in mobile sensor networks. In [22,23], the sensors are moved to optimal positions so that maximum network coverage is achieved.

Mobility is considered to be a challenge for network efficiency. However, network performance can be enhanced by the incorporation of controlled mobility. The notion of controlled mobility has been proposed so that mobility can work as an advantage in a network rather than a burden; for instance, mobile nodes with the ability to sense the energy level of a neighbor's node can change their location so as to avoid link breakages and improve the network lifetime. Natalizio et al. in [24] have illustrated the advantages and limitations of controlled mobility in mobile sensor networks. Mobility is utilized in different ways in MSNs, which can be sub-divided into networks with mobile sinks only, networks with mobile routers, and networks with all mobile nodes. Our focus is on networks where routers have the ability to move. The analytical results presented by Natalizio et al. in [25] suggest that controlled mobility of the nodes improves network performance. Nodes are moved to the most energy-efficient positions so that path lifetime of the nodes involved in a monodirectional data flow is maximized. Natalizio et al. in [26] have extended the work in [25] by considering both the forward and backward directions in a data flow. Optimal placement of the nodes is done by taking into account the energy expenditure in the two directions.

Other studies have considered controllable mobility for routing purposes. For instance, Goldenberg et al. presented a strategy in [27] in which the routers on the route are evenly spaced along a line connecting the source and destination to achieve optimal energy efficiency. Liu et al. in [28] first identify the route between the source and destination nodes, after which the router nodes can be moved to their optimal positions so as to minimize energy consumption. Loscri et al. exploit information about the nodes' positions in [29] to build the route between a source and a destination which minimizes the total traveling distance of the router nodes. Once the route is established, router nodes move to positions which are evenly spaced on the straight line connecting the source and destination. Le et al. have considered the end-to-end throughput of the network and proposed robot controllable mobility aided routing (RoCoMAR) [30]. RoCoMAR identifies the link with the lowest quality on the route and replaces it with a high-quality link through the optimal positioning of a robotic node. However, all of the abovementioned works deal with communication models such as binary or free space only and neglect the effects of multipath fading and interference on link quality. Multipath fading can often be very deep, i.e., the signals fade away completely. It also causes distortion in the radio signal and must be taken into account when developing a mobile sensor network with radio communication capabilities.

Recently, considerable attention has been given to realistic communication channels in mobile sensor and multirobot networks, with more focus on navigation strategies. Lindhe et al. in [31] proposed a strategy that improves the throughput by modifying the trajectory of the sensor nodes so that they spend more time at points with high signal strength. Zavlanos et al. in [32] defined network integrity in terms of communication rates and incorporated distributed motion control through the optimization of communication variables in a distributed hybrid multirobot network. Mostofi in [33] integrated a statistical evaluation of link quality into the motion planning function, so that each node improves its knowledge of the environment through the network's sensing capabilities and uses that knowledge for link prediction. A function incorporating the costs of both sensing and communication is used to make decisions about the motion of the nodes, and in particular to avoid deep fade spots. Seminal work on the positioning of robotic routers so as to ensure robust communication in a realistic environment has been presented by Yan and Mostofi in [34]. It is assumed that a priori information about the wireless channel is already known and can be used to predict the distribution of the channels at unvisited positions using proposed channel estimation strategies. The results show that the underlying channel properties can be predicted by randomly measuring a priori only $5 \%$ of the channel. These probabilistic channel prediction models are incorporated with motion goals for the robotic routers so as to achieve overall optimization in terms of the average end-to-end bit error rate (BER). Recently, Williams et al. in [35] developed a hybrid architecture called INSPIRE for sensing and information routing applications involving networked robots. INSPIRE utilizes two control levels, namely, the information control plane and the physical control plane, to command, control, and coordinate the robots. A simple instantiation is described in which the robotic network is dynamically reconfigured so as to ensure high-quality routes between static wireless nodes. All of the aforementioned work is not concerned with multiple access 
issues or interference among the nodes. However, interference is the main factor limiting the performance of wireless mobile sensor networks. Interference arises when the number of nodes sharing the resources is considerably large; for instance, because of the scarceness of wireless spectrum, transmission by any device at the same frequency can cause interference at the receiver [36].

\subsection{Motivation and contribution}

To summarize, the work presented in this paper is motivated by four major considerations. First, conventional routing strategies for sensor networks assume simplistic communication models $[10,11]$. Second, the papers $[31,33,34]$ that do investigate realistic communication environments do not take interference among nodes into consideration. Third, most of the previous work on realistic communication environments focuses on navigation [31] and motion control [33] in sensor networks, but does not deal with route selection or optimization. Finally, due to the complex nature of wireless channels, there are many local optimal positions and the probability of converging on a local maximum is high even if the channel has already been estimated.

Statement of contribution: We propose a communication- and position-aware reconfigurable (COMPARE) route optimization framework, with end-to-end throughput as the performance metric, by using a realistic communication model that includes noise, interference, path loss, and multipath fading in a large-scale wireless network. The contributions of this work are twofold:

- Communication-aware optimization: The optimal route with the highest throughput is identified using a communication quality-oriented route selection metric based on reception probability.

- Position-aware optimization: The optimal position with better communication quality and higher throughput for the router involved in the hop with lowest link quality is determined using particle swarm optimization (PSO). The controller is designed to move the router from its initial position to the optimal position. The channel map in general has a lot of local optimal points, and it is almost impossible to find the global optimal point directly. We show that our framework performs well by avoiding local optimal points with extremely low link quality.

\subsection{Paper outline}

The rest of this paper is organized as follows. Section 2 describes the network model, the communication link model, and the performance metric. In Section 3, we present the proposed communication- and positionaware reconfigurable route optimization strategy for large-scale MSNs, which takes into account interference, noise, path loss, and fading. The simulation and statistical results verifying the effectiveness of our framework in different scenarios are presented in Section 4. Finally, Section 5 concludes the paper.

\section{Network model}

Consider a group of mobile sensor nodes equipped with sensing, computing, and communication capabilities which are spatially distributed in a given environment and are being used to jointly perform a task. The nodes have reached their individual targets and have used their sensing capabilities to gather required information. The collected information needs to be shared with other nodes in the network. Deviations in the positions of the nodes that are small enough that the targets remain inside the sensing radii are allowed, but large deviations from the initial positions are undesirable. We use the terms robot and node interchangeably in this paper. Any node which is part of the route other than source and destination nodes is termed a router. We define the network area as a two-dimensional square in which $N$ nodes are randomly placed. When the destination node $(\mathrm{Rx})$ is significantly far from the source node (Tx), the rest of the nodes in the network act as routers by relaying information. We suppose that the nodes remain static during the period of information sharing and that any router moves only if it is required to optimize the communication throughput of the route. If there is more than one route available for data transmission from the source to the destination node, the objective is to select the route with the best throughput and to further optimize the throughput by positioning the routers so that environmental noise, path loss, fading, and interference are minimized. The solution to the problem of selecting the best route from the available routes and optimizing it by reconfiguration in a realistic communication environment involves the following steps:

- Find a suitable metric to represent the link quality in a realistic communication environment, taking into account noise, path loss, multipath fading, and interference.

- Select an optimal route on the basis of the chosen metric.

- Identify the link with the lowest throughput, as route performance is limited by that link.

- Exploit multipath fading and position information to heuristically find optimal position for the receiving router of the lowest quality link.

- Use mobility control to guide the router from the initial position to the optimal position, such that end-to-end throughput of the route is improved. 


\subsection{Communication link model}

We consider a quasi-static narrowband Rayleigh fading wireless communication link with an additive white Gaussian noise (AWGN) process and large-scale path loss exponent $\alpha$ [37]. Each transmitted signal reaches the destination via a random number of multiple paths with no dominant line of sight (LOS) signal. The received signal is corrupted by $M$ interference signals and an AWGN noise process. The variance of the noise process is denoted by $N_{o}$, and $P$ denotes the transmission power of all the nodes. The distance between the desired transmitter and receiver is denoted by $d_{D}$, and the distance between the interferer and the receiver is denoted by $d_{m}$. In the Rayleigh flat fading link model, the received power $R$ and interference power $I_{m}$ are exponentially distributed with $\bar{R}=P_{o}\left(\frac{d_{D}}{d_{o}}\right)^{-\alpha}$ and $\overline{I_{m}}=$ $P_{o}\left(\frac{d_{m}}{d_{o}}\right)^{-\alpha}$ where $d_{D}, d_{m}>d_{o}$, respectively. $d_{o}$ is the reference point located in the far field of transmit antenna, and $P_{o}$ is the average power at $d_{o}$ given as $P_{o}=P\left(\frac{\lambda}{4 \pi d_{o}}\right)^{\alpha}$. The signal-to-interference-and-noise ratio (SINR), denoted by $\zeta$, is a discrete random process given by

$$
\zeta=\frac{R}{N_{o}+I} .
$$

The SINR is factorized into signal-to-interference-ratio (SIR) and signal-to-noise-ratio (SNR). For a link between any two nodes $i$ and $j$, the SNR is the ratio of received power to noise power, given by $\zeta_{i j}=\frac{\bar{R}}{N_{o}}$, and the SIR is the ratio of received power to interference power, given as $\zeta_{m}=\frac{\bar{R}}{I_{m}}=\left(\frac{d_{D}}{d_{m}}\right)^{-\alpha}$. The cumulative density function $F(\zeta)$ for SINR is [38]

$$
F(\zeta)=1-e^{\frac{-\zeta}{\zeta_{i j}}} \cdot \prod_{m=1}^{M} \frac{1}{1+\frac{\zeta}{\zeta_{m}}}
$$

To decrease the effects of interference, a slotted ALOHA scheme is used in which, for each time slot, each node transmits independently with a certain transmission probability [39]. In Equation 1, $I$ is the accumulated interference power at the receiver given by $I=\sum_{m=1}^{M} B_{m} I_{m}$. The transmission probability $p_{t}$ is assumed to follow a Bernoulli distribution so that $B_{m}$ is a sequence of independent Bernoulli distributed random variables with $\mathbb{P}\left(B_{m}=1\right)=p_{t}$ and $\mathbb{P}\left(B_{m}=0\right)=1-p_{t}$.

\subsection{Link quality estimation using reception probability}

The quality of a wireless communication link is determined by the instantaneous SINR $(\zeta)$ between two nodes. In general, outage probability [40] is used to estimate the link quality and is defined as the probability that the instantaneous $\operatorname{SINR}(\zeta)$ is below a certain threshold $\zeta_{t}$.
Accordingly, a packet will be successfully received if $\zeta \geq$ $\zeta_{t}$. The probability that the instantaneous SINR between two generic nodes $i$ and $j$ is above the threshold $\zeta_{t}$ is called reception probability (RP), denoted by $p_{r}:=\mathbb{P}\left[\zeta_{i j} \geq \zeta_{t}\right]$. The value of $\zeta_{t}$ depends upon the modulation and coding scheme [41]. RP has been used previously for link quality estimation in wireless sensor networks. Flushing et al. have used the packet reception probability to estimate link quality in [42] and refer to it as mobility-assisted proactive probing and learning estimates (MAPPLE). Alizai et al. [43] and Becher et al. [44] have also used the predicted probability of successful packet transmission to estimate link quality. The RP for a slotted ALOHA scheme with a Rayleigh fading channel is calculated by using Equation 2, and [45] shows that it can be factorized into reception probabilities of noise-only $p_{r}^{N}$ and interference-only $p_{r}^{I}$ networks given as

$$
\begin{aligned}
p_{r}:= & \mathbb{P}\left[\zeta_{i j} \geq \zeta_{t}\right]=1-F(\zeta)=\exp \left(-\frac{\zeta_{t}}{\zeta_{i j}}\right) \cdot \prod_{m=1}^{M} \frac{1}{1+\frac{\zeta_{t}}{\zeta m}}, \\
= & \exp \left(-\frac{\zeta_{t} N_{o}}{P_{o}\left(\frac{d_{D}}{d_{o}}\right)^{-\alpha}}\right) \\
& \cdot \prod_{m=1}^{M}\left\{\mathbb{P}\left(B_{m}=1\right) \cdot \frac{1}{1+\frac{\zeta_{t}}{\zeta m}}+\mathbb{P}\left(B_{m}=0\right)\right\}, \\
= & \exp \left(-\frac{\zeta_{t} N_{o}}{P_{o}\left(\frac{d_{D}}{d_{o}}\right)^{-\alpha}}\right) \cdot \prod_{m=1}^{M}\left(\frac{p_{t}}{\left.1+\zeta_{t}\left(\frac{d_{D}}{d_{m}}\right)^{\alpha}+1-p_{t}\right)}\right) \\
= & \underbrace{\exp \left(-\frac{\zeta_{t} N_{o}}{P_{o}\left(\frac{d_{D}}{d_{o}}\right)^{-\alpha}}\right)}_{p_{r}^{N}} \underbrace{\prod_{m=1}^{M}\left(1-\frac{p_{t} \zeta_{t}}{\zeta_{t}+\left(\frac{d_{m}}{d_{D}}\right)^{\alpha}}\right)}_{p_{r}^{I}} .
\end{aligned}
$$

The basic concepts of framework are reinforced by means of a series of MATLAB simulations. The RP is investigated by simulating the behavior of $p_{r}^{N}$ as a function of SNR threshold and relative distance between two nodes as shown in Figure $2 \mathrm{a}$. The $p_{r}^{N}$ decreases as the distance between the nodes increases and is inversely proportional to the SNR threshold. The narrowband Rayleigh fading wireless communication channel with an AWGN process and path loss exponent $\alpha$ is simulated using MAT$\mathrm{LAB}$, with a view to understand the relationship between the number of paths and the RP. Figure $2 \mathrm{~b}$ shows the relationship between $p_{r}^{N}$ and the number of paths on the link between the nodes. The RP decreases as the number of paths increases between two nodes. The values assigned to the parameters in the simulations are listed in Table 1.

\subsection{Performance measure}

An appropriate QoS performance measure needs to be defined for a route optimization framework in mobile sensor networks. For instance, throughput is a conventional estimate for the amount of traffic delivered by the network $[8,13]$. We define the normalized throughput as 


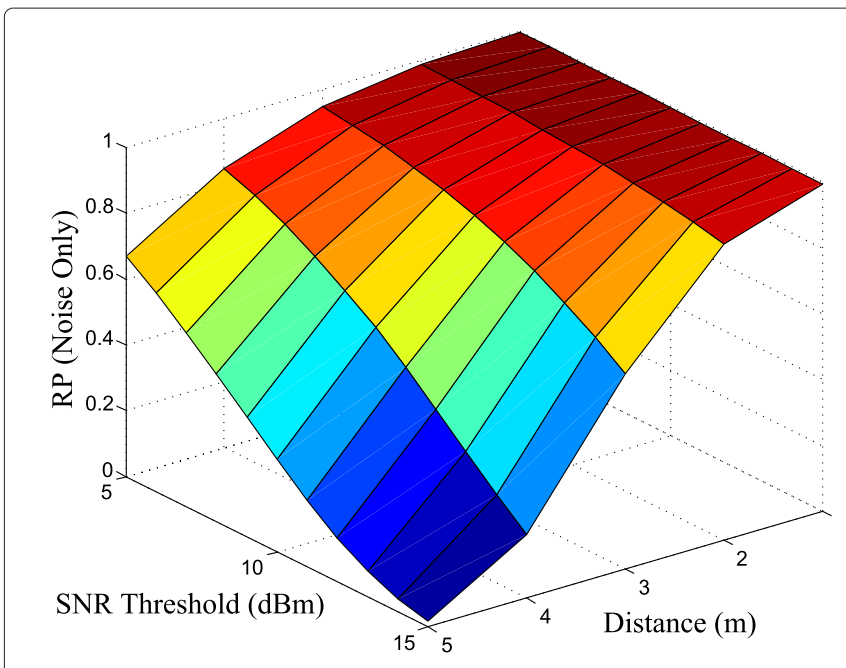

(a) Distance (1-5m), SNR threshold (5-15 $\mathrm{dBm})$

Figure 2 Reception probability (noise only).

the expected number of successful packet transmission for a given node per time slot [37]. This normalized throughput can be thought of as the fraction of time a channel is utilized and is measured in Erlangs. We will regard end-to-end throughput over a multihop connection as the performance measure for a route. The end-to-end throughput for a route is defined as the minimum of throughput values of the links involved in constituting the route. The optimal route configuration is the one with the highest possible end-to-end throughput. The throughput between two generic nodes $i$ and $j$ is given by

$$
T P_{i j}=p_{t}\left(1-p_{t}\right) \times p_{r_{i j}},
$$

where $p_{r}$ is the RP as given in Equation 3, $p_{t}$ is the probability that node $i$ transmits, and $\left(1-p_{t}\right)$ is the probability that node $j$ does not transmit in the same time slot. The probability of transmission $p_{t}$ in each time slot depends on the number of interferers on that particular link. The maximum throughput achievable by a network using slotted ALOHA is 0.3679 , i.e., slotted ALOHA provides a maximum channel utilization of 0.3679 Erlangs [37].

Table 1 Values for parameters used in simulation

\begin{tabular}{lcc}
\hline Parameter & Description & Value \\
\hline$P$ & Transmit power & $0 \mathrm{dBm}$ \\
$N_{0}$ & Noise variance & $-85 \mathrm{dBm}$ \\
$\zeta_{t}$ & SINR threshold & $10 \mathrm{dBm}$ \\
$\lambda$ & Wavelength & $0.12 \mathrm{~m}$ \\
$\alpha$ & Path loss exponent & 4 \\
$N$ & Total nodes & 150 \\
$k_{1}$ and $k_{2}$ & Controller parameters & 0.2 and 1 \\
\hline
\end{tabular}

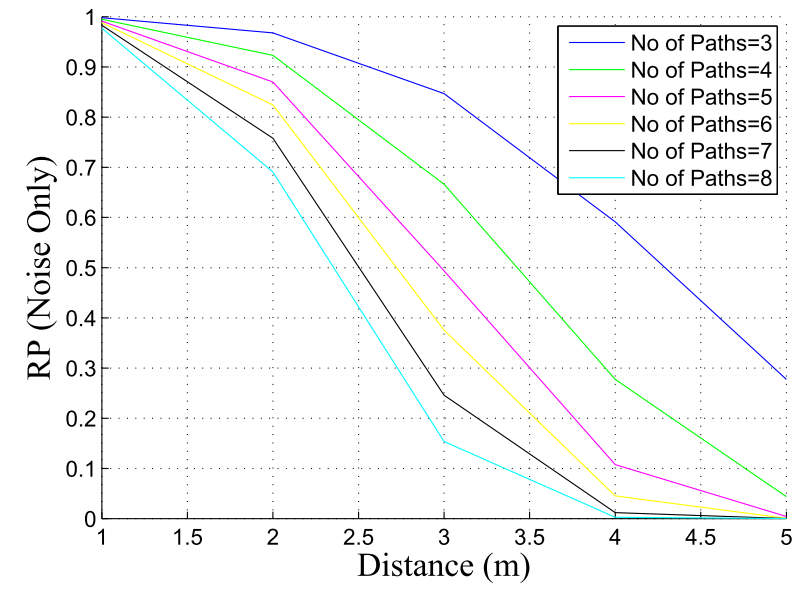

(b) Number of paths (3-8)

\section{COMPARE route optimization}

The COMPARE framework is comprised of three main components, i.e., communication-aware route selection, link quality estimation, and position-aware optimization. These components allow the routes to be selected and optimized by appropriately positioning a particular router as depicted in Figure 3. Whenever the source

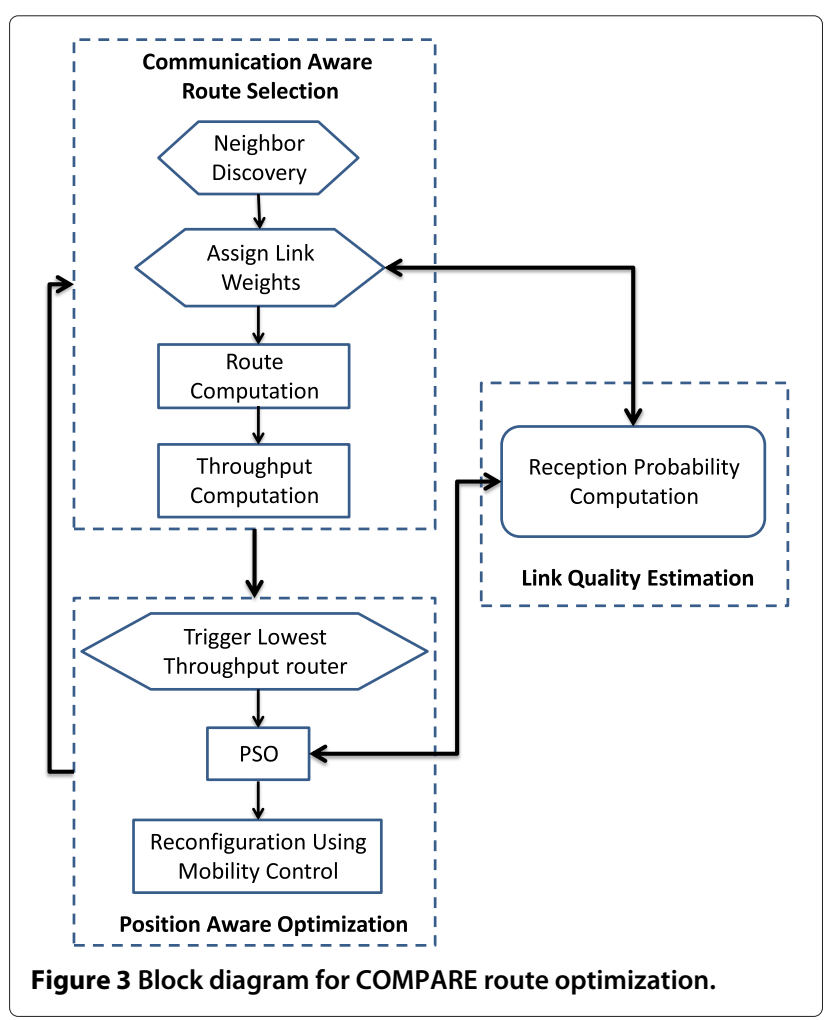


node requires a route to the destination node, the communication-aware route selection (CARS) phase is called upon. First, each node in the network performs neighbor discovery for the initialization of an ad hoc wireless network. Second, weights based on the RP are assigned to the individual links a node has with each of its neighbors. The link quality estimation entity is used for the computation of RP between any two nodes. Third, Dijkstra's algorithm is applied to identify the route with the highest RP. The last step in CARS is to compute the end-to-end throughput of the overall route. A detailed explanation of CARS is provided in Section 3.1. When communication-aware route selection is complete, position-aware optimization (PAO) is called to perform route reconfiguration and throughput optimization. The receiving router on the link with the lowest throughput is triggered by the destination node. The router uses particle swarm optimization and link quality estimation to find a new position with higher throughput. Once the new position is computed, the mobility controller is used to move the particular router to that optimal position. The CARS phase is recalled to compute the overall throughput for the reconfigured route. The PAO is described in more detail in Section 3.2.

\subsection{Communication-aware route selection}

An important requirement of mobile sensor networks is to route traffic from a source to a destination node. To achieve this, we need to determine a route using routing algorithm. The communication-aware route selection incorporates the routing decisions with knowledge of communication links including noise, path loss, multipath fading, and interference. We show how end-to-end throughput of route depends on the quality of individual links and route selection. The routing schemes essentially rely on efficient shortest path algorithms such as the Bellman-Ford [46] or Dijkstra algorithm [47] to compute the optimal route. These algorithms find routes with the minimum weight as determined by the given route selection metric.

In order to incorporate noise, path loss, fading, and interference, we use the route selection metric based on RP presented in Equation 3 and find the route with maximum RP. As presented in Algorithm 1, if a route is required for the data transmission, all the nodes identify their one hop neighbors and compute RP and achievable TP. The communication links are independent and does not affect each other's probability. Once RP for all links in the network is computed, we take the negative logarithm to turn multiplication of probabilities into addition of non-negative link costs. The Dijkstra algorithm uses these link costs to compute the minimum cost routes, which correspond to the routes with maximum RP. The Dijkstra algorithm have both centralized and distributed versions. In centralized versions, all route choices are made at a central node and routes are broadcast to the rest of network. While in the distributed version [48], the computation of route is shared among the network nodes with information exchanged between them. All the nodes calculate shortest paths to each other based on the received link cost information.

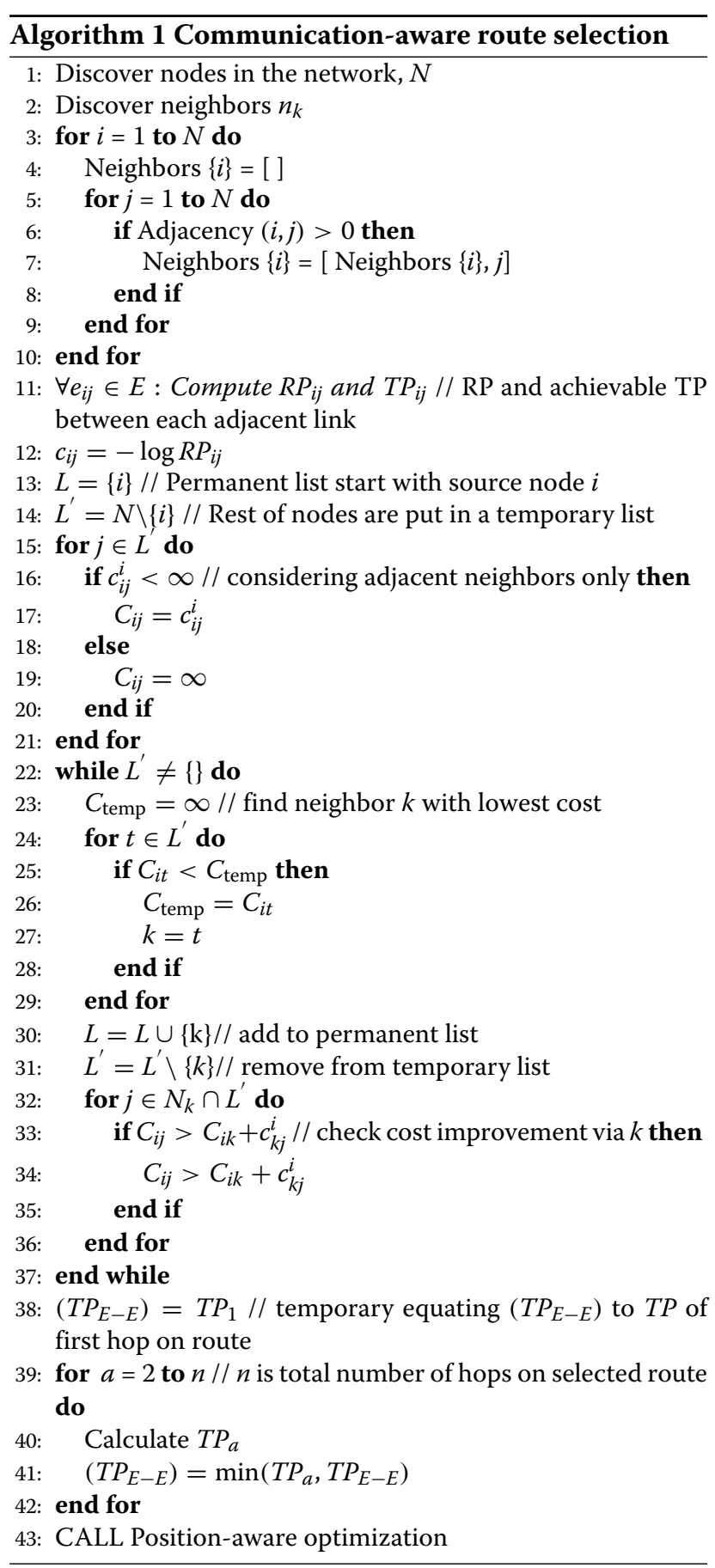


It is assumed that each node knows the position of its neighbors using either the Global Positioning System (GPS) or a localization technique for MSNs [49]. Even if the position information is obtained using a centralized system such as the GPS, each node locally calculates the RP for its own adjacent links using Equation 3. The mobile sensor network is represented as a directed graph $G(N, E)$, where $N$ is the set of nodes and $E$ is the set of links between nodes, called edges. The link between any two nodes $i$ and $j$ is denoted by a weighted edge $e_{i, j} \neq e_{j, i}$. In case of communication-aware route selection, the cost of edge is equal to the negative logarithm of its respective RP. We consider two generic source and destination nodes, labeled as $i$ and $j$, respectively, in a network containing $N$ nodes. $c_{i j}$ is the link cost between nodes $i$ and $j$, while $C_{i j}$ is the cost of minimum-cost route between nodes $i$ and $j$. The complete set of nodes $N$ is divided into two lists: the nodes already considered are stored in permanent list $L$, and nodes not yet considered are stored in temporary list $L^{\prime}$. It is observed from Algorithm 1 that list $L$ expands while $L^{\prime}$ shrinks as the algorithm progresses and nodes are deleted from $L^{\prime}$ and added to $L$. The algorithm stops if $L^{\prime}$ is empty. Algorithm 1 finds optimal routes to all the destination nodes in the network. In case route to a specific destination $j$ is required instead of routes to all destinations, an IF and exit operation 'if $(k=j)$, exit' is added in between lines 29 and 30. The essence of Algorithm 1 lies in the way it expands the list $L$ and computes the shortest paths to nodes that are neighbor of nodes of list $L$ but are not yet in the list. As presented in Algorithm 1, on each iteration, list $L$ is expanded by including a neighboring node $k$ of node $i$ with the minimum link cost. In line 33 , neighboring nodes of $k$ are examined at each iteration to see if there are any changes in the minimum cost from the last iteration. The original shortest path is kept by the algorithm if there is no improvement in cost. An identifier is used to track the next hop from source node $i$ to the destination node $j$. The end-to-end throughput $\left(T P_{E-E}\right)$ is determined in a distributed manner. Initially, $\left(T P_{E-E}\right)$ is set to be the throughput of the first hop on the route, and its value is compared with throughput of the second hop. The minimum of the two throughputs is assigned as the temporary value of $\left(T P_{E-E}\right)$. This process is repeated for all the hops on the route unless finalized $\left(T P_{E-E}\right)$ information is obtained at the destination node.

\subsection{Position-aware optimization}

An environment with multipath fading and interference generates signal strength variations over distances of a wavelength. Small changes in the position of the router can be exploited to improve the received signal strength [50]. In this phase, we exploit the information we have about the multipath fading and positions of the transmitter and interfering nodes to improve the endto-end throughput of the selected route. Assuming that the fading channel is learned beforehand, the link quality at the unvisited location can be predicted using the position information of the transmitter and interferers of that link. Even if the channel is known beforehand, the stochastic nature of a wireless channel makes it difficult for iterative methods to find the global optimal position, as the channel map in general has a lot of local optimal positions and is unpredictable due to noise, path loss, multipath fading, and interferences. In such a situation, heuristic-based algorithms are good candidates to achieve beneficial performance in avoiding extreme local optima. Previously, the PSO-based algorithm incorporated with distributed virtual force algorithm (VFA) is developed by Loscri et al. in [51] to improve dynamic event coverage for mobile sensor networks. We use the PSO to find the optimal position for the router, so that the RP and overall throughput are improved.

Once the route is selected in first phase of COMPARE, the destination node triggers the receiving router on link with the lowest throughput which is limiting the overall route performance. The router executes the PSO algorithm and provides its current position as input to PSO. PSO along with link estimation generates a new position for the router with a better RP and higher throughput. The route is reconfigured by moving the router to the optimal position using feedback mobility control. In order to ensure that relocation of the router results in improved throughput and does not diminish the overall throughput, we return to the communication-aware route selection phase so as to identify the throughput of the optimal available route incorporating the new router position.

\subsubsection{PSO-based optimal position search}

PSO is a heuristic optimization technique which models a set of potential problem solutions as a swarm of particles moving about in a virtual search space [52]. The solution is called a particle, and a group of particles is referred to as a population. The PSO finds the optimal position for the router in three steps as shown in the flow diagram in Figure 4: (i) Initialization: A random population matrix is generated with each row representing a candidate solution. Each particle in the population begins the search at a random position $\left(X_{i, j}\right)$ and with a random velocity $\left(V_{i, j}\right)$ in the $n$-dimensional search space. Here, $i$ represents the particle index and $j$ represents the dimension in the search space. Prospective solutions are optimized as the particles are attracted to the positions that yield the best individual results, and each particle remembers the position of best performance $\left(P_{i, j}\right)$. (ii) Evaluation: The individual best performances are compared and the best of all particles is identified using the reception probabilities calculated from Equation 3. (iii) Adaptation: Finally, the 


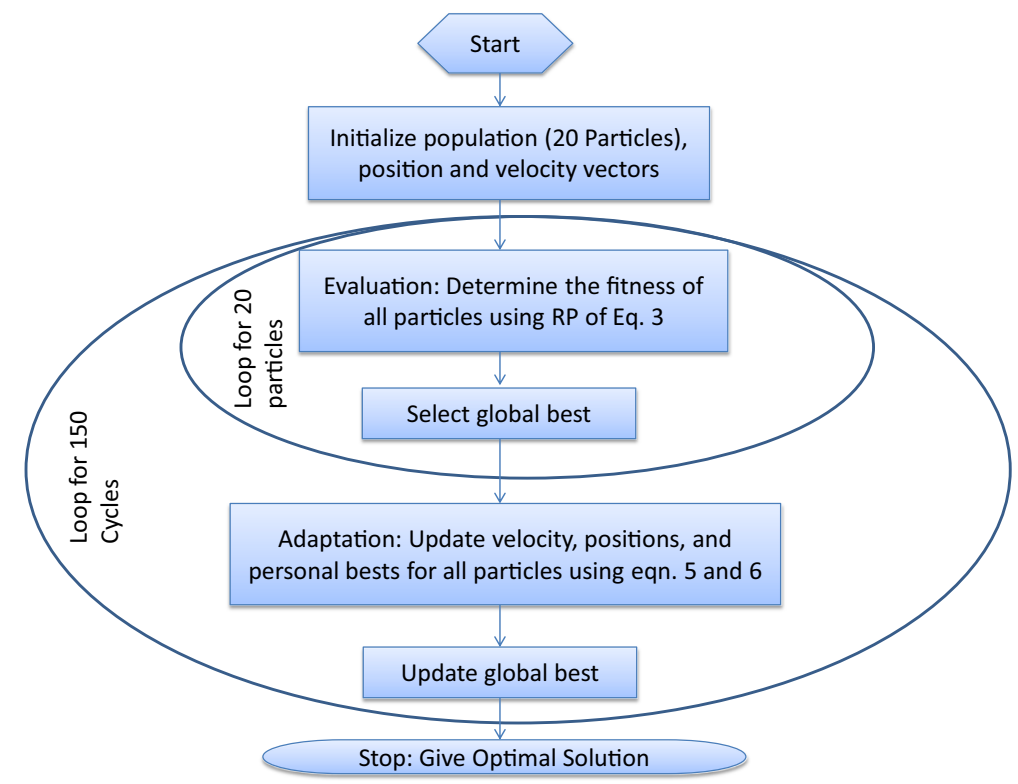

Figure 4 Flow chart for PSO-based optimal position search.

particles are updated by assigning a velocity to each element using Equations 5 and 6 . The particle that achieves the best performance in terms of RP is called the global particle.

$$
\begin{gathered}
V_{i j}=\underbrace{V_{i j}}_{\text {Inertia }}+\underbrace{\alpha_{1} \cdot\left(P_{i j}-X_{i j}\right)}_{\text {Personal influence }}+\underbrace{\alpha_{2} \cdot\left(p g(i)-X_{i j}\right),}_{\text {Social influence }} \\
X_{i j}(n)=X_{i j}(n-1)+\alpha_{3} \tanh \left(V_{i j}\right),
\end{gathered}
$$

where $V_{i j}$ is the velocity and $X_{i j}$ is the position of each particle, $P$ is the individual best performance, and $p g$ is the global best performance in the neighborhood. The inertia term in Equation 5 keeps the particle moving in approximately the same direction and with the same velocity; the personal influence term helps to improve the best individual performance for each particle by making the particle move towards its best individual position; and the social influence term makes the particle move in the direction of its best neighbor. $\alpha_{1}, \alpha_{2}$, and $\alpha_{3}$ are uniformly distributed random numbers generated between $(0,1)$.

In our problem, we integrate evaluation of the communication quality with PSO; the RP in Equation 3 is used as a fitness function for PSO. In multipath fading, the channel quality varies with small change in positions; therefore, the distances between the positions of the particles is kept small enough to search in the local vicinity of the receiving node such that the new position is not far from the old position. The initial particle elements are randomly generated in the range $(0,1)$. We use a swarm of 20 particles and 150 cycles. In each cycle, the algorithm reevaluates the previous best positions and compares the new fitness value based on RP with the previous ones to get a more accurate measure of the actual fitness. By running the algorithm in this fashion, the learning accuracy is significantly improved. The nature of the problem requires a smooth transition from one position to another, so the hard limit for the max and min velocities is replaced with a smoothing tanh function [53]. PSO generates a new position with improved RP and throughput. This position is given to the feedback mobility controller, which moves the router to the desired optimal position.

\subsubsection{Feedback mobility control of mobile agents}

Once the optimal position is computed using the PSO, the mobile router needs to move to that position. Our objective is to design a control law so that the mobile router moves smoothly and arrives at exactly the desired position. Before formulating the control design [54], we present the differential equations that directly control the acceleration of the router,

$$
\begin{aligned}
& \dot{x_{1}}=x_{2}, \\
& \dot{x_{2}}=u,
\end{aligned}
$$

where, $x_{1}, x_{2}$, and $u$ represent the position, velocity, and control input, respectively. The initial position will be the position of the mobile router after the optimal route has been identified using the communication-aware route selection algorithm. The state space representation of the above equation is

$$
\begin{aligned}
& \dot{x}=A x+B u, \\
& A=\left[\begin{array}{ll}
0 & 1 \\
0 & 0
\end{array}\right], x=\left[\begin{array}{ll}
x_{1} & x_{2}
\end{array}\right]^{T}, B=\left[\begin{array}{ll}
0 & 1
\end{array}\right]^{T},
\end{aligned}
$$


where $x$ represents the state vector of the router, $A$ is the system matrix, $B$ is the actuator matrix, and $u$ is the control law,

$$
u=-K x .
$$

Since the system has two states, so $K=\left[\begin{array}{ll}k_{1} & k_{2}\end{array}\right]$ is $1 \times 2$ row vector. Combining Equations 9 and 10 results in

$$
\dot{x}=(A-B K) x .
$$

The appropriate selection of $k_{1}$ and $k_{2}$, will move the router to the target location.

\subsection{Computational complexity analysis}

The proposed COMPARE framework consists of multiple algorithms with different complexities. Due to this, we need to analyze the computational complexity of the individual algorithms used in the framework. CARS utilizes the simplest implementation of the Dijkstra algorithm for optimal route selection. The implemented version in our problem utilizes an ordinary array, and the computational complexity for communication-aware route selection using an ordinary array is $O\left(N^{2}\right)$.

The position-aware optimization is based mainly on particle swarm optimization, which is only executed on the router with the lowest link quality to search for the optimal location where the router contributes better to the end-to-end throughput. In terms of intercommunication through the network, searching for the optimal location is independent to searching for the most optimal route and throughput computation. The computational complexity of PSO depends on the number of initial particles and the number of cycles to reach the global optimum [55]. The computational complexity for the PSO is given as $O(M X)$. In our case, the number of particles is $M=20$ and the number of cycles is $X=150$. If the network is large enough, $O(M X)$ is minimal as compared to $O\left(N^{2}\right)$. Therefore, the computational complexity of the COMPARE algorithm is considered as $O\left(N^{2}\right)$ approximately.

\section{Performance evaluation}

The objective of the simulation is to evaluate the effectiveness of the proposed communication- and positionaware reconfigurable route optimization framework. Several tests have been performed involving different scenarios to demonstrate the robustness and correctness of the proposed framework. First, we quantify the benefits of incorporating a realistic communication model with routing decisions. The quality of realistic links is characterized by the RP, and the RP-based metric is used for route selection. The end-to-end throughput performance of the RP metric is compared with conventional metrics such as the ED and $\mathrm{HC}$ in a multipath fading channel with noise, path loss, and interference. Second, we show how the throughput of the route selected by the RP-based metric can be further optimized by reconfiguring that particular route. Using PSO, we find an optimal position for the receiving router of minimum throughput link and then move the router to that position using the mobility control $(\mathrm{MC})$. The throughput performance of the reconfigured route (RP-PSO-MC) is compared with the original route computed using the RP-based metric.

\subsection{Simulation setup}

The values assigned to the fixed parameters are shown in Table 1. The values selected for the parameters are in accordance with real-world low-power wireless networks [56].

\subsubsection{Assumptions}

The following operational assumptions underlie the development of the proposed framework.

- Each node has a unique identification (ID).

- Every node knows the relative distance to its neighboring nodes.

- A priori channel information is available.

- The transmission power is the same for all nodes.

- Single-path routing is considered, and only the route chosen and optimized by the COMPARE framework is used for data transmission from the source to the destination.

\subsection{Results from illustrative scenarios}

We check the correctness and validity of the proposed COMPARE framework in different network scenarios, i.e., sparse, dense, and mixed up. A total of $N=150$ nodes are placed with a Gaussian random distribution within the $50 \times 50$ square test area. The test area is divided into cells or subareas with size $d \times d$. In our problem, we consider three scenarios, with the differences between them depending on the spatial proximity of the nodes to each other. The sparse scenario is defined as a network with only one node in each subarea; the dense scenario has four nodes in each subarea; and the mixed-up scenario has two nodes in each subarea.

For each scenario, we validate the proposed approach by evaluating the simulation results for two separate cases, i.e., one-to-one and one-to-all. The one-to-one simulation focuses on a single route in order to perform a hop-by-hop analysis of the selected route and examines the process by which the router on the link with limited throughput is optimally positioned. In the one-to-all case, we test the performance of COMPARE for different kinds of routes with varying numbers of hops, and levels of interference and distance. We collect statistical data for 149 routes and choose the median instead of the mean as a descriptor of the data because the distribution is quite skewed. The dispersion of the data is measured using the interquartile 
range (IQR). The IQR is defined as the difference between 25 th and 75 th percentiles of the data.

\subsubsection{Scenario 1: sparse network}

One-to-one. We first investigate the case when a source node wants to share information with a specific distant destination node in a sparse network. The network scenario is shown in Figure 5, where a randomly selected source node-1 requires a route to the destination node- 72 . The routes are selected using three route selection metrics based on HC, ED, and RP.

Figure 6a shows the achievable end-to-end throughput of the routes selected by the different metrics. The RPbased route outperforms the HC- and ED-based metrics in terms of end-to-end throughput. The throughput gain for the RP-based route is $32 \%$ and $78 \%$ more than the EDand $\mathrm{HC}$-based routes, respectively. Figure $6 \mathrm{~b}$ shows the number of hops traversed by each metric. Intuitively, the $\mathrm{HC}$ metric chooses the route with the lowest number of hops, i.e., 14, but in realistic communication scenarios, minimizing the hop count does not necessarily increase the end-to-end throughput. The RP-based metric takes four extra hops to reach the destination, but it incorporates the stochastic nature of the wireless links and chooses the link with the highest reception probability, resulting in higher throughput. By contrast, the ED- and $\mathrm{HC}$-based schemes select the route irrespective of the conditions and nature of the wireless links. As expected, the ED-based scheme choose the route with the smallest physical distance between the source and destination, as shown in Figure 6c.

The RP-based route is further optimized using the position-aware optimization phase. First, the link with the minimum throughput inside the route is identified. In the case of scenario 1 , the link between router-112 and router-120 on the RP-based route has the lowest reception probability, i.e., 0.345 , and an individual throughput of 0.0261 , limiting the overall route performance. The PSO is used by router- 120 to find a new position, so that the reception probability and throughput are improved. The RP-based route is reconfigured as the router moves to the new position using MC. The yellow edges in Figure 7 represent the link connectivity of router-120 at the new position computed by the PSO. The initial position of router-120 in the RP-based route is $(29.65,28.89)$. After implementing position-aware optimization, router120 moves to $(27.70,28.09)$. Thus, moving the router by a small distance of $209 \mathrm{~cm}$ at an angle of 0.385 achieves a gain in the end-to-end throughput of $32 \%$, compared to the previous throughput of the RP-based route as shown in Figure 8a. The RP-PSO-MC represents the reconfigured route after PSO and MC have been applied to the route selected using the RP metric. Figure $8 \mathrm{~b}, \mathrm{c}$ shows that the number of hops and the Euclidean distance remain the same for both RP- and RP-PSO-MC-based routes in this particular case.

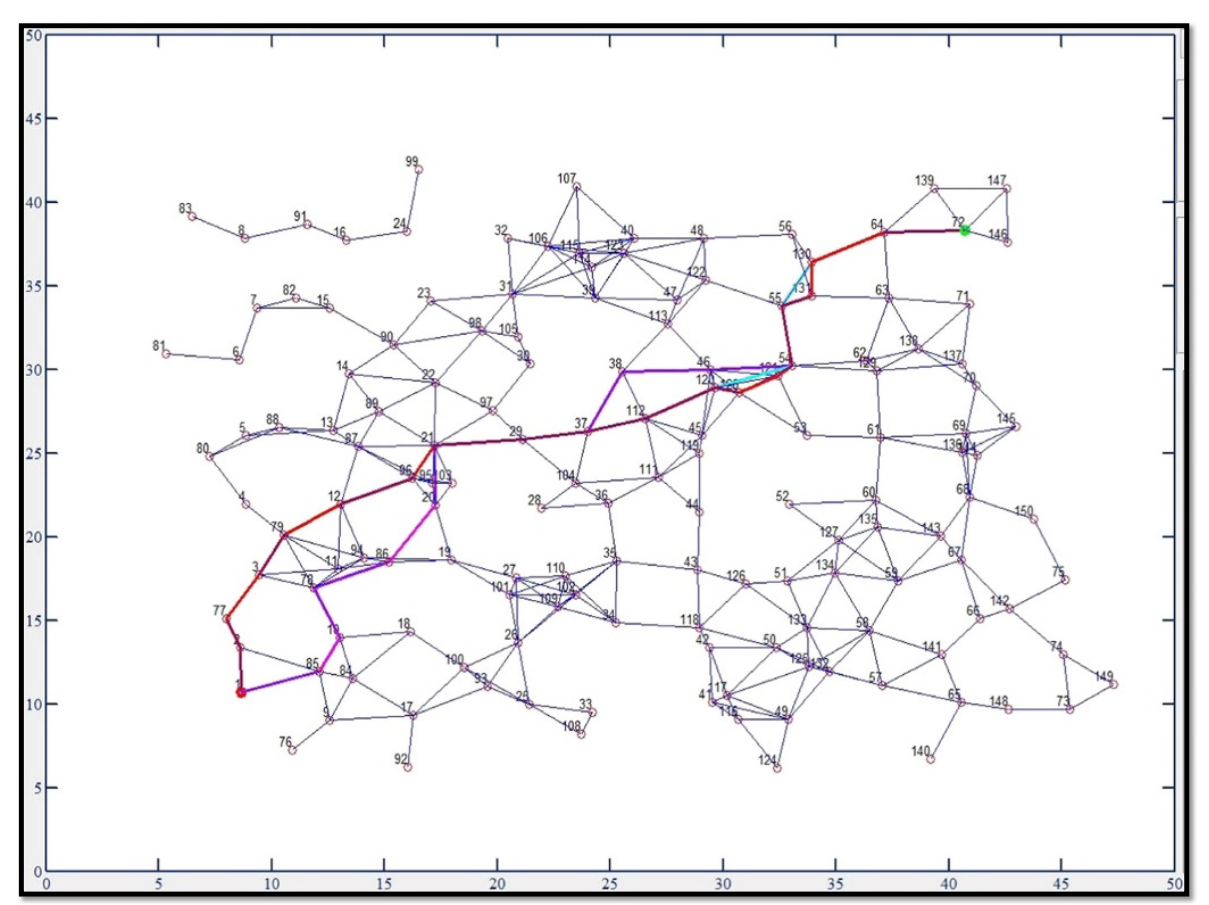

Figure 5 A sparse network of 150 nodes with routes selected from source node-1 (red) to destination node-72 (green). Using HC (magneta)-, ED (cyan)-, and RP (red)-based metrics. 


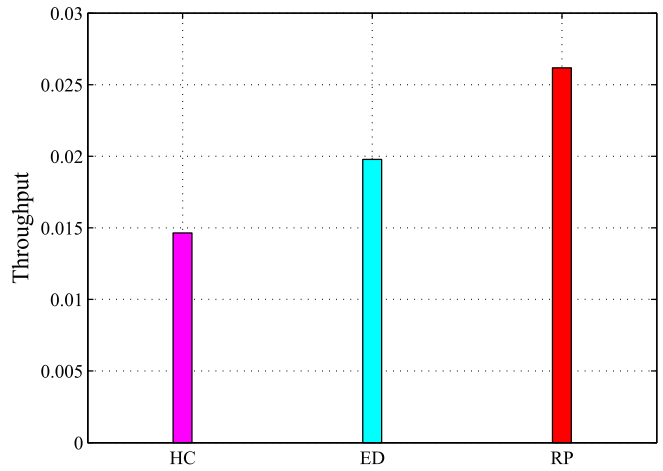

(a)

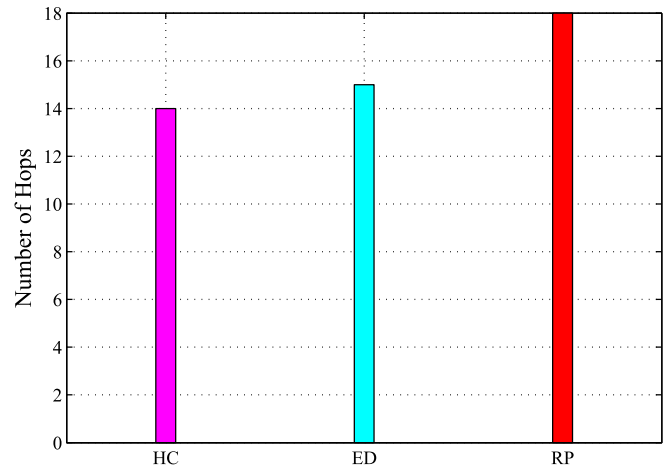

(b)

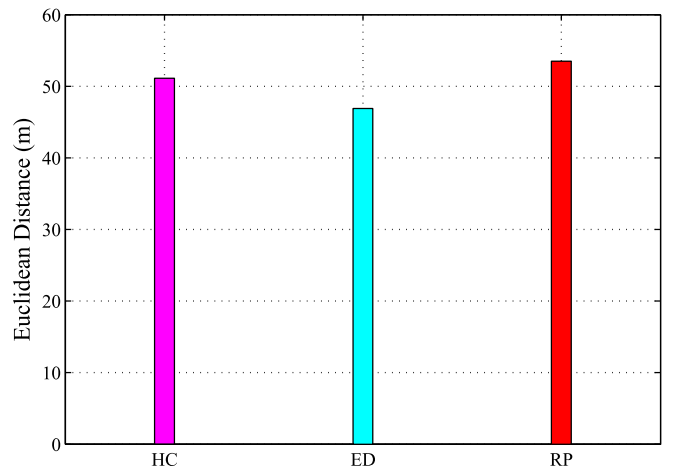

(c)

Figure 6 Throughput (a), number of hops (b), and Euclidean distance (c) of routes for a single source-destination pair in sparse network. Using HC-, ED-, and RP-based metrics.

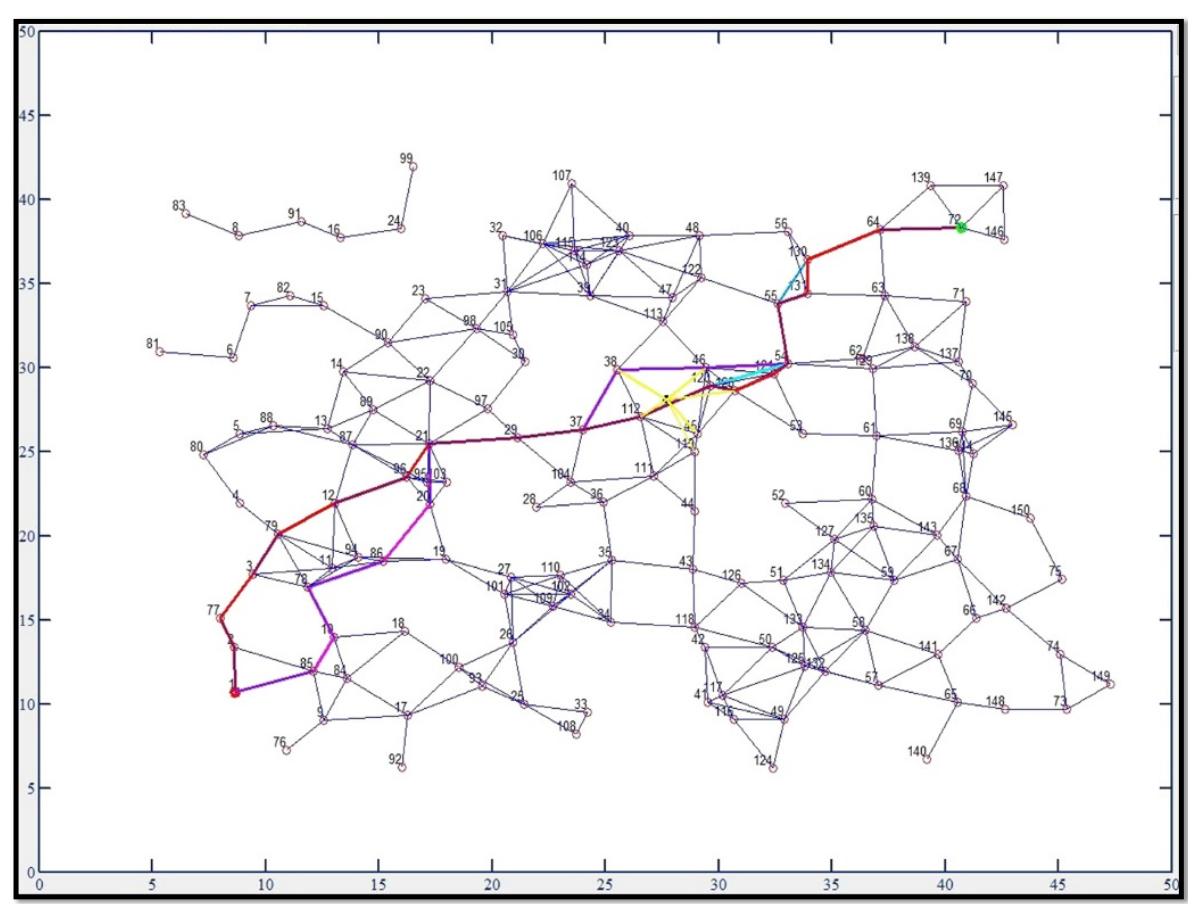

Figure $7 \mathrm{~A}$ sparse network of 150 nodes with yellow edges depicting the connectivity of relocated router. 


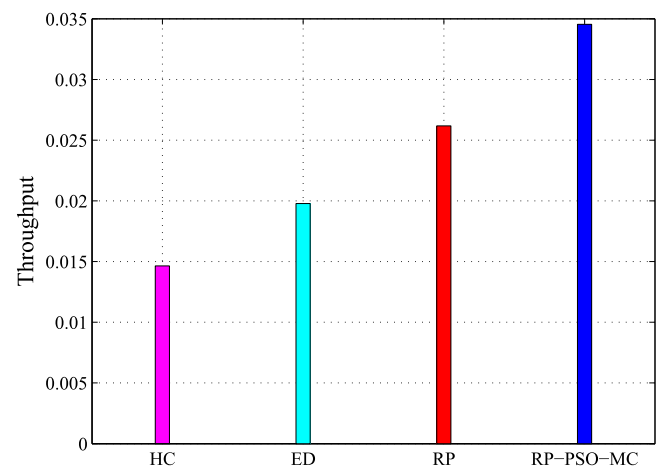

(a)

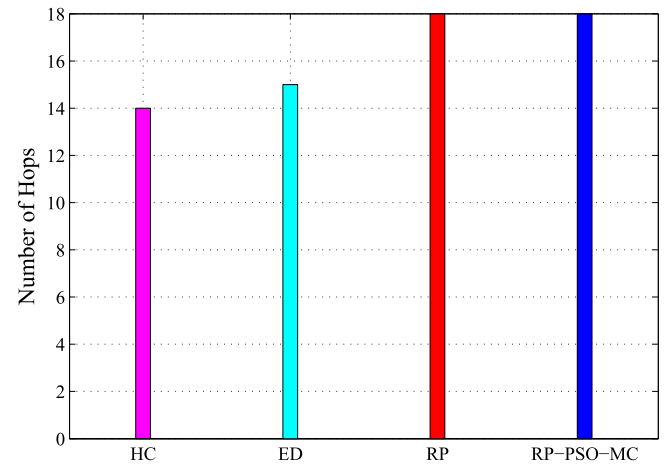

(b)

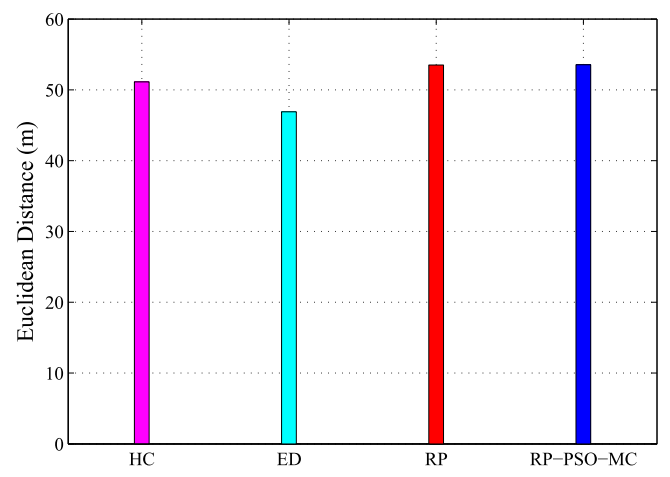

(c)

Figure 8 Throughput (a), number of hops (b), and Euclidean distance (c) for a single source-destination pair. Using HC-, ED-, RP-, and RP-PSO-MC-based schemes in sparse network.

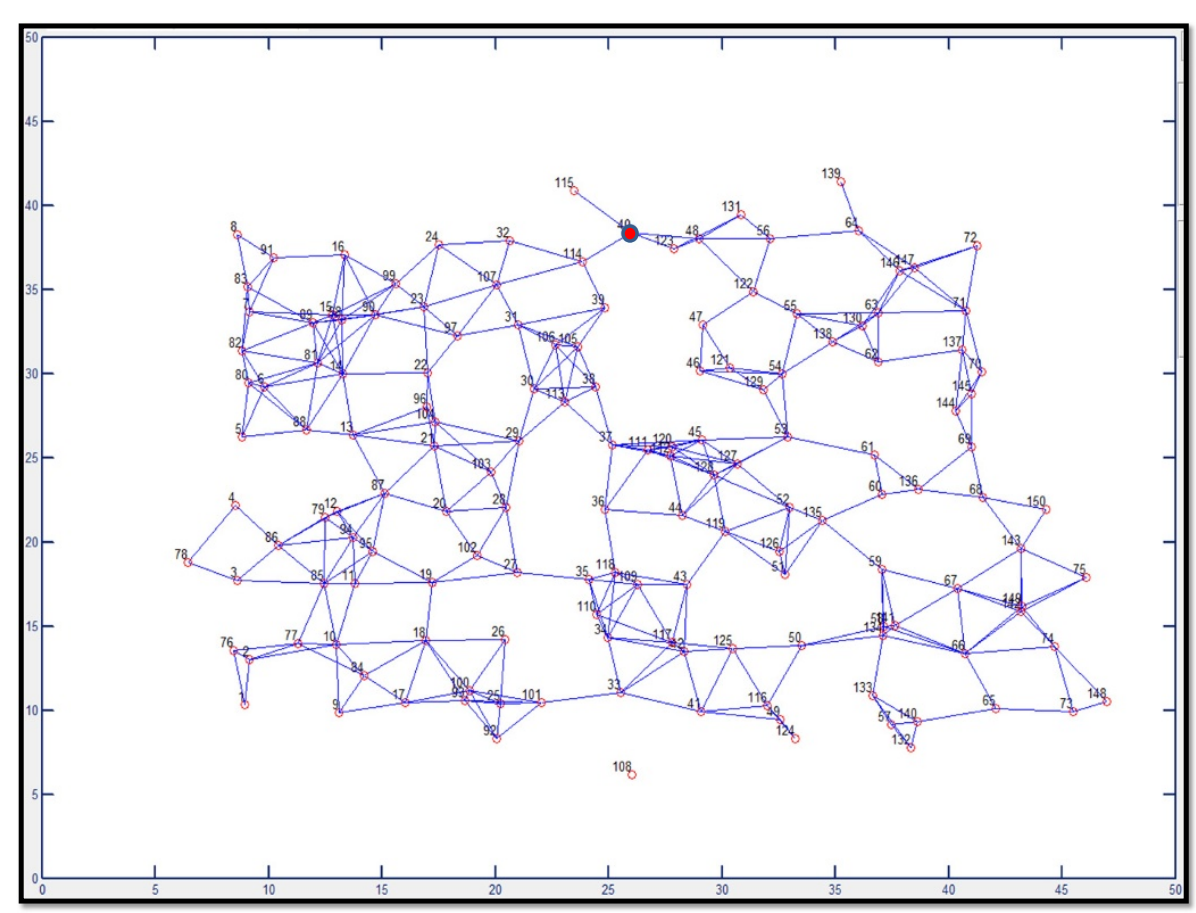

Figure 9 A sparse network of 150 nodes with source node-40. Routes to all other destinations are selected using HC-, ED-, RP-, and RP-PSO-MC-based schemes. 
One-to-all. In order to further validate the proposed route optimization framework, we consider a situation in which a source node requires routes to all the other nodes in the network. In the scenario shown in Figure 9, node-40 is randomly selected as the source node and routes to all the other destinations are computed using the HC-, ED-, and RP-based metrics. Furthermore, each route selected by the RP-based metric is reconfigured using positionaware optimization and mobility control (RP-PSO-MC). Figure 10a shows the statistical properties of the end-toend throughput for the 149 source-destination pairs. The performance of the proposed COMPARE scheme is validated again, as it achieves a median throughput gain of $19 \%$ compared to the RP-based route without positionaware optimization. The median number of hops and the Euclidean distance remain the same in this particular case, as shown in Figure10b,c, respectively.

Intuitively, it seems that the reception probability is higher at small distances, but in reality, it can be the reverse, as the link quality also depends on interference and fading. The reception probability can be very low even at small distances if there is high interference and deep fades. The scheme we are proposing generates routes with a higher number of hops than the conventional metrics, but manages to generate optimized throughput by exploiting multipath fading and position information.

\subsubsection{Scenario 2: dense network}

One-to-one: In the dense scenario shown in Figure 11, node- 1 and node-147 are randomly chosen as the source and destination nodes. The routes are selected using the HC-, ED-, RP-based metrics, and the link between router $-104 \rightarrow 144$ is identified as the link with the lowest throughput on the RP-based route. Router-144 is moved to a new position using position-aware optimization, and the yellow lines show the links of router-144 at the new position.

Figure 12a illustrates the end-to-end throughput for the HC-, ED-, RP- and RP-PSO-MC-based routes. Reconfiguration of the RP-based route by moving router-144 by a short distance of $241 \mathrm{~cm}$ at an angle of 0.718 achieves a gain of $14 \%$ compared to the previous route. The previous

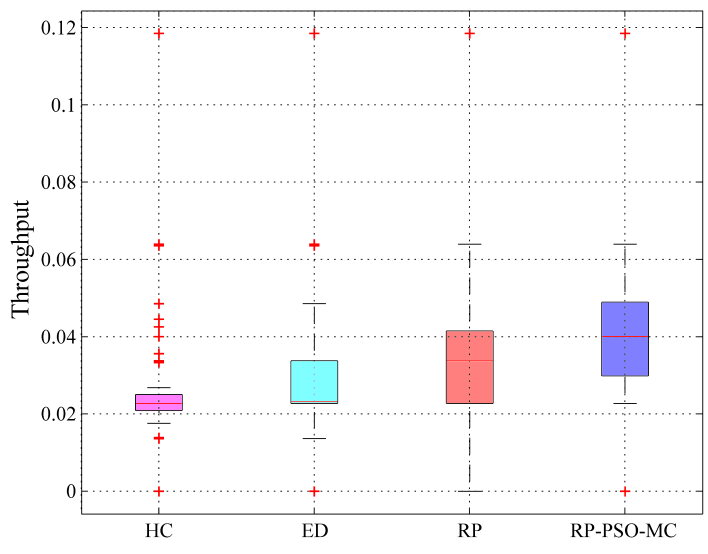

(a)

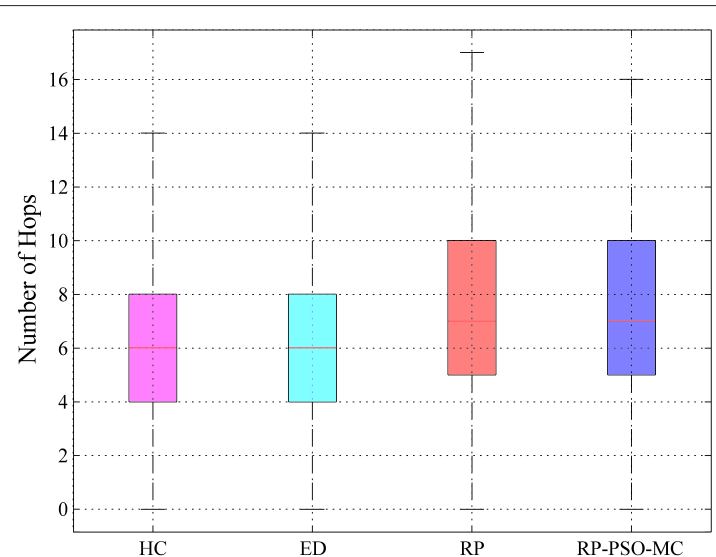

(b)

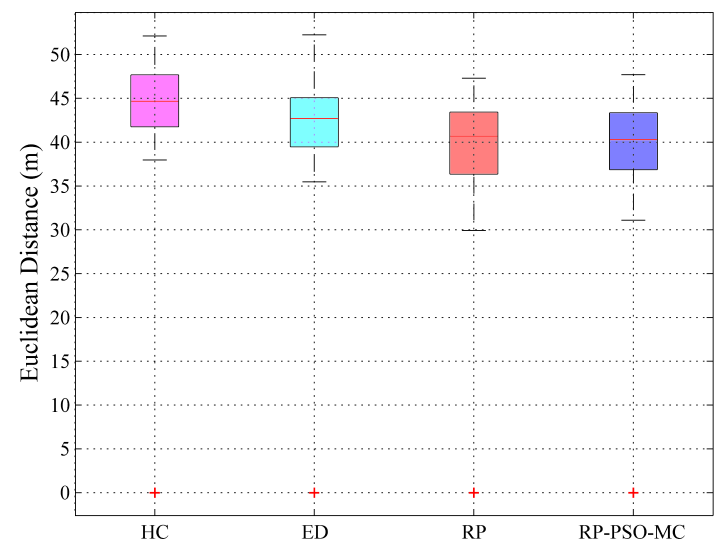

(c)

Figure 10 Throughput (a), number of hops (b), and Euclidean distance (c) for routes of 149 source-destination pairs in a sparse network. 


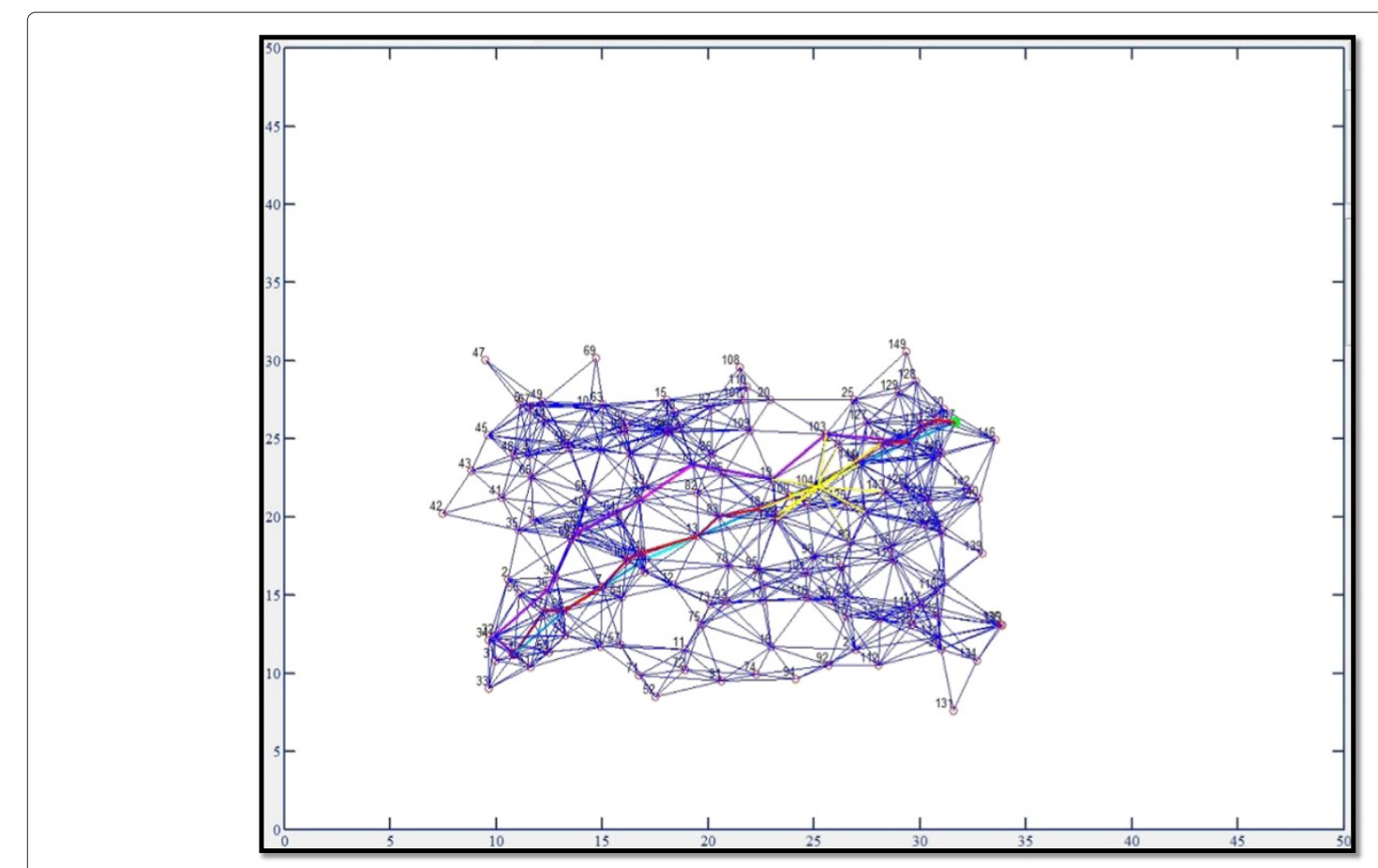

Figure 11 A dense network of 150 nodes with routes selected from source node-1 (red) to destination node-72 (green). Using HC (magneta)-, ED (cyan)-, and RP (red)-based metrics.

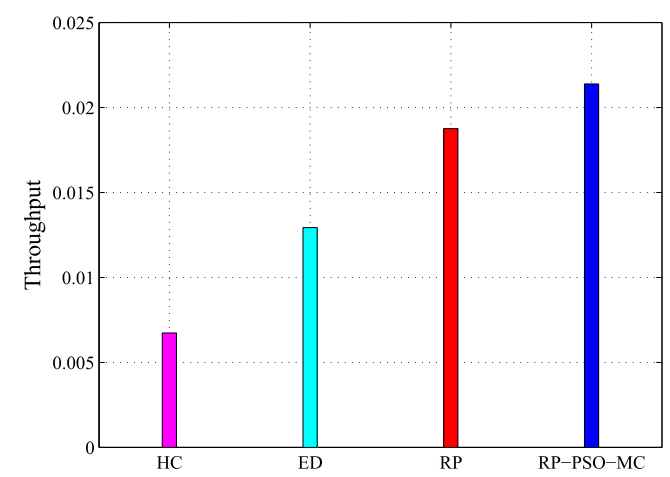

(a)

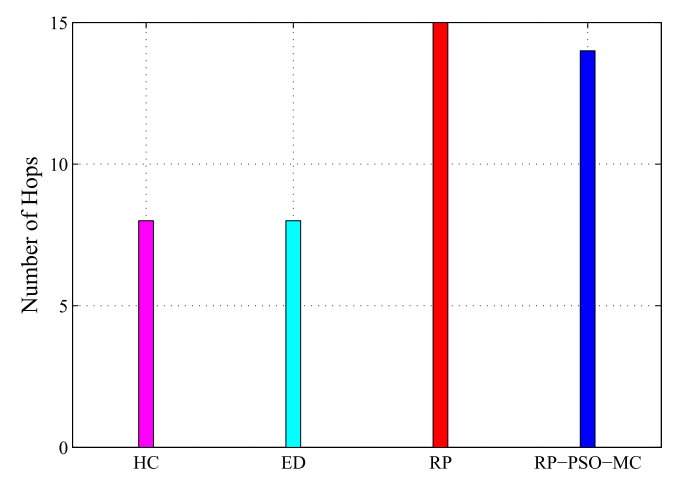

(b)

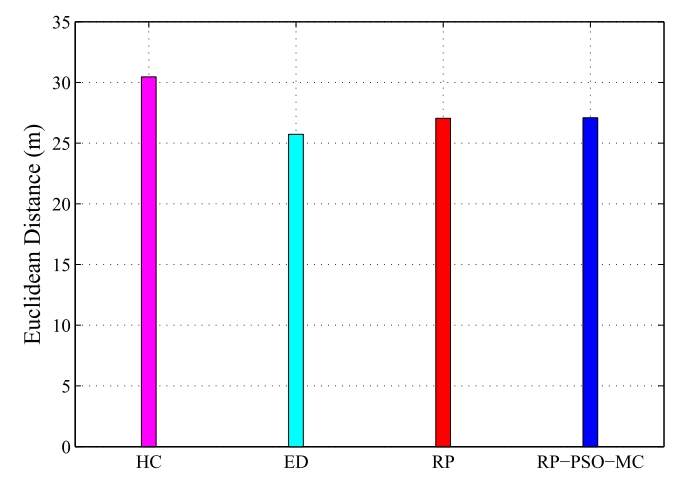

(c)

Figure 12 Throughput (a), number of hops (b), and Euclidean distance (c) for a single source-destination pair. Using HC-, ED-, RP-, and RP-PSO-MC-based schemes in a dense network. 
Table 2 RP- and RP-PSO-MC-based selected routes

\begin{tabular}{ll}
\hline Scheme & Route \\
\hline $\mathrm{RP}$ & $1 \rightarrow 37 \rightarrow 56 \rightarrow 7 \rightarrow 80 \rightarrow 81 \rightarrow 13 \rightarrow 83 \rightarrow 18 \rightarrow$ \\
& $100 \rightarrow 104 \rightarrow 144 \rightarrow 145 \rightarrow 124 \rightarrow 130 \rightarrow 150 \rightarrow 147$ \\
RP-PSO-MC & $1 \rightarrow 37 \rightarrow 56 \rightarrow 7 \rightarrow 80 \rightarrow 81 \rightarrow 13 \rightarrow 83 \rightarrow$ \\
& $18 \rightarrow 104 \rightarrow 144 \rightarrow 145 \rightarrow 124 \rightarrow 130 \rightarrow 150 \rightarrow 147$ \\
\hline
\end{tabular}

and new positions of the router are $(27.03,23.54)$ and (25.20, 21.96), respectively. In a dense network, the COMPARE framework reduces the number of hops in the reconfigured route, as shown in Figure $12 \mathrm{~b}$ where the RPPSO-MC-based route has one less hop than the simple RP-based route.

The routes selected by the RP and RP-PSO-MC schemes are shown in Table 2 . It is observed that before reconfiguration, the $\mathrm{RP}$-based route follows the hops $18 \rightarrow 100 \rightarrow 104$; when router- 144 is moved to the optimal position using position-aware optimization, the route is reconfigured and establishes a direct link with router18 , i.e., $18 \rightarrow 100$. Minimizing the number of hops in the route has a positive impact on the performance of a sensor network; it helps by decreasing delay and increasing robustness.

One-to-all: The proposed framework is also tested for the one-to-all case in a dense scenario. Node- 1 is randomly selected as the source in Figure 11, and the routes to all the other nodes in the network are computed using the HC, ED, RP, and RP-PSO-MC schemes. Figure 13a shows a statistical comparison of the throughputs for the 149 routes. The median throughput gain for the RPPSO-MC routes is $20 \%$ compared to the simple RP-based routes. The overall number of hops is also minimized once the RP-based routes have been reconfigured, as shown in Figure 13b. The Euclidean distance is the lowest for the ED-based metric and is almost the same in the RP- and RP-PSO-MC-based routes, as illustrated in Figure $13 \mathrm{c}$.

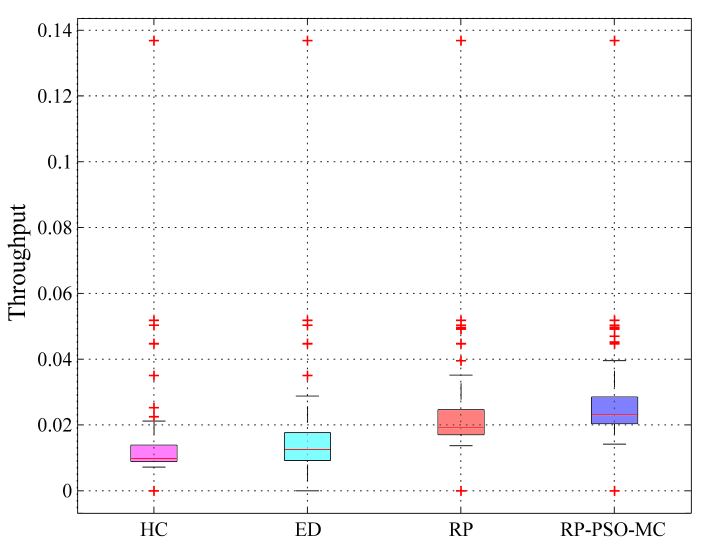

(a)

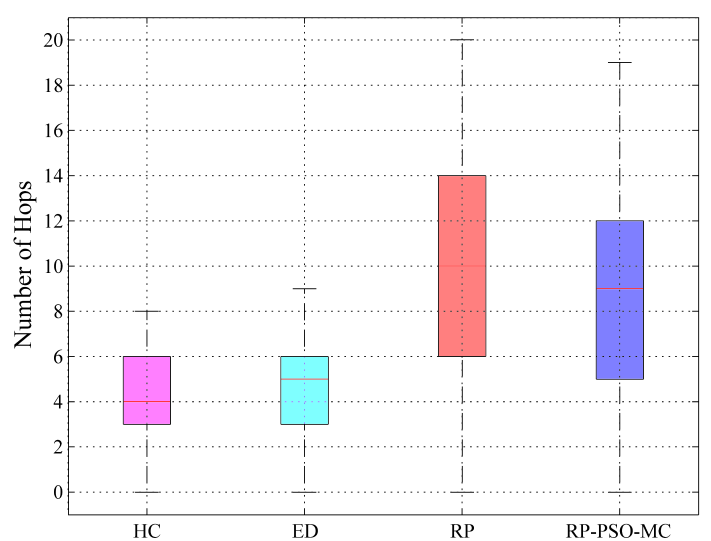

(b)

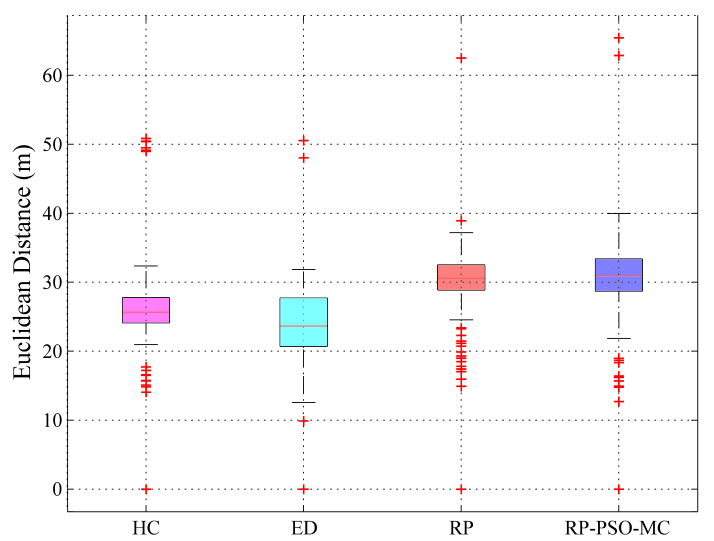

(c)

Figure 13 Throughput (a), number of hops (b), and Euclidean distance (c) for routes of 149 source-destination pairs in a dense network. 


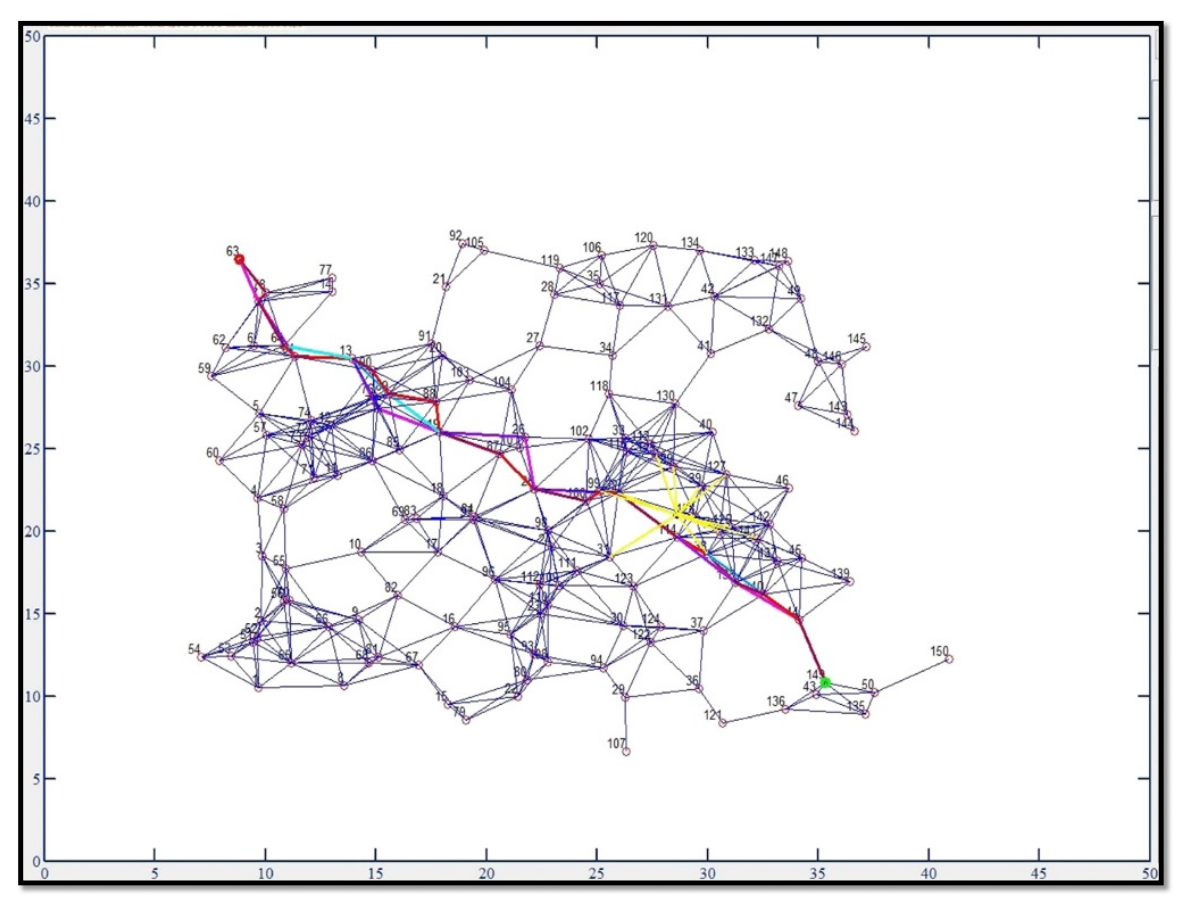

Figure 14 A mixed-up network of 150 nodes with routes selected from source node-1 (red) to destination node-72 (green). Using HC (magneta)-, ED (cyan)-, and RP (red)-based metrics.

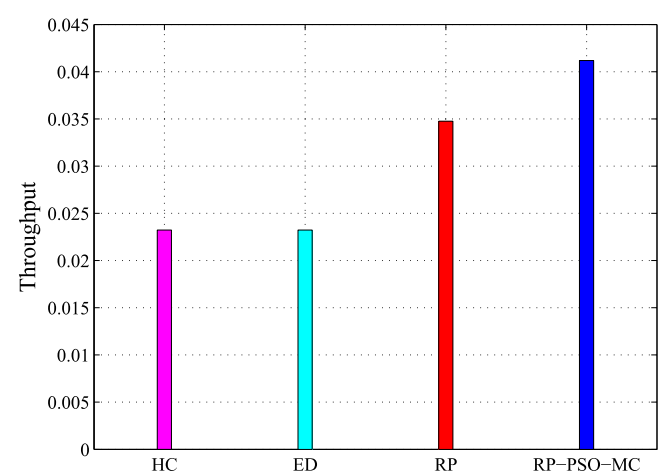

(a)

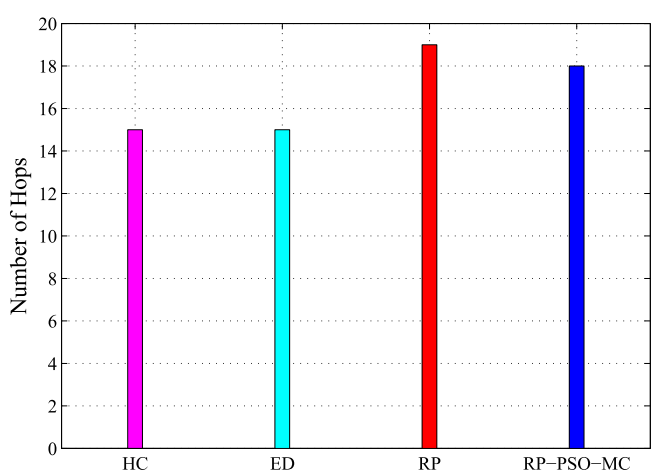

(b)

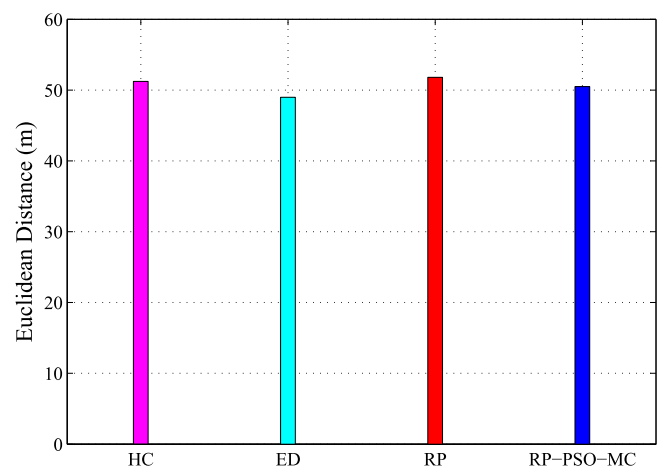

(c)

Figure 15 Throughput (a), number of hops (b), and Euclidean distance for a single source-destination pair. Using HC-, ED-, RP-, and RP-PSO-MC-based schemes in a mixed-up network. 


\subsubsection{Scenario 3: mixed-up network}

One-to-one: In this simulation, we consider a scenario in which some parts of the network are densely populated while others are sparsely connected, as shown in Figure 14. Initially, the routes are selected on the basis of the HC-, ED-, and RP-based metrics, and the RP-based route is further optimized by reconfiguring the position of the router with the lowest link quality. In Figure 14, the link $32 \rightarrow 114$ is limiting the overall performance of the RP-based route. Router-114 is moved to its optimal position using PSO and MC, and the yellow edges represent the links of router-114 at the optimal position. Figure 15a shows that the RP-PSO-MC-based route provides a higher throughput compared with the simple RP-based route. This gain is achieved through only a small change in position, as the position for router- 114 in the RP-based route is $(28.61,19.62)$ and the optimal position computed by PSO is $(28.61,21.00)$. Thus, router- 114 is moved only $137 \mathrm{~cm}$ at an angle of 1.567 to achieve an $18 \%$ throughput gain. The number of hops for the reconfigured route is also less than for the RP-based route, demonstrating the usefulness of the proposed scheme, as illustrated in Figure 15b. Figure $15 \mathrm{c}$ shows that the ED-based route traverses the least distance and the Euclidean distances for the other schemes are approximately the same.

One-to-all: The routes from source node- 1 to all the other nodes in Figure 14 are computed using the same procedure as described in the previous scenarios. A statistical analysis of the throughputs for 149 routes is shown in Figure 16a. The RP-PSO-MC-based routes achieve a median throughput gain of $31 \%$ compared to the original RP-based routes. The number of hops for the RP-PSOMC-based routes is less than in the RP-based routes, as illustrated in Figure 16b. The Euclidean distances for the routes based on the RP and RP-PSO-MC schemes are approximately the same, as shown in Figure 16c.

\section{Conclusions}

In a mobile sensor network, it is crucial to achieve a high throughput for ongoing data transmission between

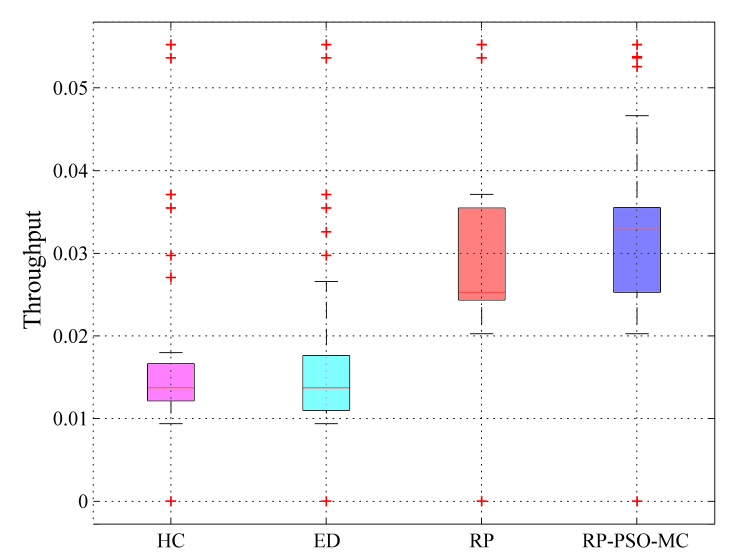

(a)

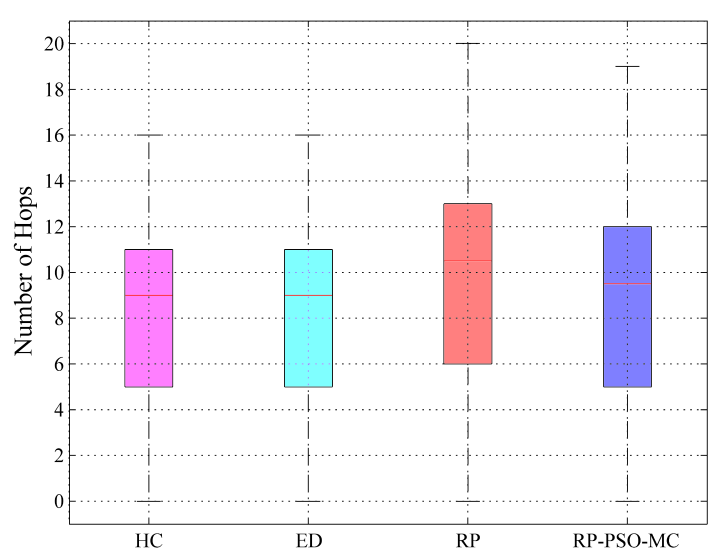

(b)

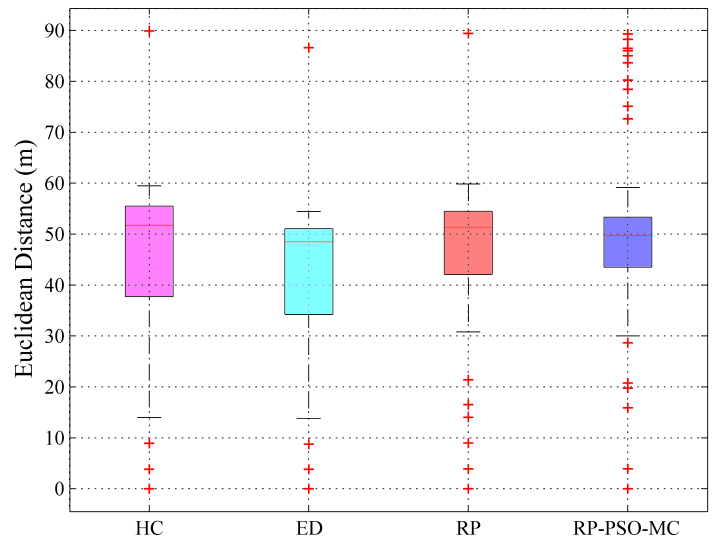

(c)

Figure 16 Throughput (a), number of hops (b), and Euclidean distance (c) for routes of 149 source-destination pairs in a mixed-up network. 
two far away nodes connected by a number of router nodes. To this end, we have proposed a COMPARE route optimization framework. The performance of the proposed framework has been examined in the presence of noise, path loss, multipath fading, and interference. Reception probability is used to estimate link quality and select the route with a higher end-to-end throughput. The throughput of the selected route is further optimized by reconfiguring it in the position-aware optimization phase. The single-path routing is considered, and only the route selected by the COMPARE framework is used for data transmission. The throughput performance of COMPARE was compared with the conventional route selection metrics through simulation. We showed that by exploiting the multipath fading, mobility, and position information of nodes, COMPARE has resulted in considerably better performance.

Currently, we are investigating coordinative reconfigurable route optimization using the proposed framework. In addition, we considered the single-route data transmission in this paper which has limited fault tolerance; the performance of the proposed framework in a multiple route infrastructure is of potential interest for future work.

\section{Competing interests}

The authors declare that they have no competing interests.

\section{Authors' contributions}

AF designed the overall study, implemented the framework for simulation, defined scenarios to validate the simulator, conducted the experiments and analysis, and prepared the draft. Ml contributed to the implementation of position-aware optimization and in the preparation of the final manuscript. TDN provided technical oversight, supervised the overall work, and revised the manuscript. All authors contributed to the discussion and revision of the manuscript. All authors read and approved the final manuscript.

\section{Acknowledgements}

Special thanks to Malcolm Anderson for his proofreading of this paper. This research is supported in part by the University Research Grant at the University of Brunei Darussalam under Grant No. UBD/PNC2/2/RG/1.

Received: 23 May 2014 Accepted: 24 November 2014

Published: 2 December 2014

\section{References}

1. O Tekdass, V Isler, in IEEE International Conference on Robotics and Automation (ICRA). Robotic routers (IEEE, Pasadena, CA, 2008), p. $1513-1518$

2. MM Zavlanos, GJ Pappas, Potential fields for maintaining connectivity of mobile networks. IEEE Trans. Robot. 23(4), 812-816 (2007)

3. MCD Gennaro, A Jadbabaie, in 45th IEEE Conference on Decision Control. Decentralized control of connectivity for multi-agent systems (San Diego, CA, 2006), p. 3628-3633

4. Y Yan, Y Mostofi, in Military Communications Conference (MILCOM). Robotic router formation: a bit error rate approach (IEEE, San Jose, CA, 2010), pp. 1287-1292

5. Z Butler, D Rus, Event based motion control for mobile sensor networks. Pervasive Computing. IEEE. 2(4), 34-42 (2003)

6. M Grossglauser, DNC Tse, Mobility increases the capacity of ad hoc wireless networks. IEEE/ACM Trans. Netw. 10(4), 477-486 (2002)

7. G Nmeth, ZR Turnyi, A Valk, Throughput of ideally routed wireless ad hoc networks. ACM Mobile Comput. Commun. Rev. 5(4), 40-46 (2001)
8. P Gupta, PR Kumar, The capacity of wireless networks. IEEE Trans. Inf. Theory. 46(2), 388-404 (2000)

9. DA Maltz, J Broch, DB Johnson, Lessons from a full-scale multihop wireless ad hoc network testbed. IEEE Pers. Commun. 8(1), 8-15 (2001)

10. DB Johnson, DA Maltz, Dynamic Source Routing in Ad-Hoc Wireless Networks. Mobile Computing. (Springer, US, 1996), pp. 153-181

11. VD Park, MS Corson, in Proceedings of the INFOCOM'97. Sixteenth Annual Joint Conference of the IEEE Computer and Communications Societies. Driving the Information Revolution. INFOCOM'97. A highly adaptive distributed routing algorithm for mobile wireless networks (IEEE Computer Society, Washington, DC, USA, 1997), p. 1405

12. M Mauve, J Widmer, $\mathrm{H}$ Hartenstein, A survey on position-based routing in mobile ad hoc networks. IEEE Netw. Mag. 15(6), 30-39 (2001)

13. DeDSJ Couto, D Aguayo, J Bicket, R Morris, in 9 th Annual International Conference on Mobile Computing and Networking (MobiCom). MobiCom '03. A high-throughput path metric for multi-hop wireless routing (ACM, San Diego, 2003), pp. 134-146

14. J Chen, R Linemail, Y Liemail, Y Sunemail, LQER : a link quality estimation based routing for wireless sensor networks. Sensors. 8(2), 1025-1038 (2008)

15. K Sohrabi, J Gao, V Ailawadhi, G Pottie, Protocols for self-organization of a wireless sensor network. IEEE Pers. Commun. 7(5), 16-27 (2000)

16. K Akkaya, M Younis, Energy and QoS aware routing in wireless sensor networks. Cluster Comput. 8(2-3), 179-188 (2005)

17. A Fida, DH Pham, NJ Tuah, T Ngo, in Proceedings of the 13th International Conference on Intelligent and Autonomous Systems. IAS'13. Communication aware route selection in wireless sensor networks (Springer, Padova, Italy, 2014)

18. Y Kim, M Mesbahi, On maximizing the second smallest eigenvalue of a state-dependent graph laplacian. IEEE Trans. Automatic Control. 51(1), 116-210 (2006)

19. C Dixon, EW Frew, in Proceedings of the 1st International Conference on Robot Communication and Coordination. RoboComm '07. Maintaining optimal communication chains in robotic sensor networks using mobility control (IEEE Press, Piscataway, 2007), pp. 1-118. http://dl.acm.org/ citation.cfm?id=1377868.1377870

20. KXu, H Hassanein, G Takahara, Q Wang, Relay node deployment strategies in heterogeneous wireless sensor networks. IEEE Trans. Mobile Comput. 9(2), 145-159 (2010)

21. W Wang, V Srinivasan, KC Chua, Extending the lifetime of wireless sensor networks through mobile relays. IEEE Trans. Netw. 16(5), 1108-1120 (2008)

22. Y Zou, K Chakrabarty, Sensor deployment and target localization in distributed sensor networks. ACM Trans. Embedded Comput. Syst. (TECS). 3(1), 61-91 (2004)

23. G Wang, K Cao, TL Porta, Movement-assisted sensor deployment. IEEE Trans. Mobile Comput. 5(6), 640-652 (2006)

24. E Natalizio, V Loscrí, Controlled mobility in mobile sensor networks: advantages, issues and challenges. Telecommunication Syst. 52(4), 2411-2418 (2013)

25. E Natalizio, V Loscrí, E Viterbo, Optimal placement of wireless nodes for maximizing path lifetime. IEEE Commun. Lett. 12(5), 362-364 (2008)

26. E Natalizio, V Loscri, F Guerriero, A Violi, Energy spaced placement for bidirectional data flows in wireless sensor network. Commun. Lett. IEEE. 13(1), 22-24 (2009)

27. DK Goldenberg, J Lin, AS Morse, BE Rosen, YR Yang, in 5th ACM International Symposium on Mobile Ad Hoc Networking and Computing. Towards mobility as a network control primitive (Tokyo, Japan, 2004), pp. 163-174

28. H Liu, A Nayak, I Stojmenovic, in 3rd International Conference on Mobile Ad-Hoc and Sensor Networks. Localized mobility control routing in robotic sensorwireless networks (Beijing, China, 2007), pp. 19-31

29. V Loscri, E Natalizio, C Costanzo, Simulations of the impact of controlled mobility for routing protocols. EURASIP J. Wireless Commun. Netw. 7(1), 1687-1472 (2010)

30. DV Le, H Oh, S Yoon, RoCoMAR: robots' controllable mobility aided routing and relay architecture for mobile sensor networks. Sensors. 13(7), 8695-8721 (2013). doi:10.3390/s130708695

31. M Lindhe, $\mathrm{KH}$ Johansson, in IEEE International Conference on Robotics and Automation (ICRA). Adaptive exploitation of multipath fading for mobile sensors (Alaska, USA, May 2010), pp. 1934-1939 
32. MM Zavlanos, A Ribeiro, GJ Pappas, Network integrity in mobile robotic networks. IEEE Trans. Automatic Control. 58(1), 3-18 (2013)

33. Y Mostofi, in IEEE International Conference on Robotics and Automation, ICRA. Communication-aware motion planning in fading environments (California, USA, May 2008), pp. 3169-3174

34. Y Yan, Y Mostofi, Robotic router formation in realistic communication environments. IEEE Trans. Robot. 28(4), 810-827 (2012)

35. A RK Williams, B Gasparri, in International Conference on Intelligent Robots and Systems (IROS), IEEE. Krishnamachari, Route swarm: Wireless network optimization through mobility (Chicago, USA, 2014), pp. 3775-3781

36. M Haenggi, RK Ganti, Interference in large wireless networks. Foundations Trends in Netw. 3(2), 127-248 (2008)

37. T Rappaport, Wireless Communications: Principles and Practice, 2nd edn (Prentice Hall PTR, Upper Saddle River, NJ, USA, 2001)

38. M Haenggi, in International Symposium on Circuits and Systems (ISCAS), vol. 5. Towards a circuit theory for sensor networks with fading channels (IEEE, Vancouver, BC, 2004), pp. 908-911

39. R Nelson, L Kleinrock, The spatial capacity of a slotted aloha multihop packet radio network with capture. IEEE Trans. Commun. 32(6), 684-694 (1984)

40. M Simon, M Alouini, Digital Communication over Fading Channels, 2nd edn. (John Wiley and Sons, Hoboken, NJ, USA, 2005)

41. A Ephremides, Energy concerns in wireless networks. IEEE Trans. Wireless Commun. 9(4), 45-59 (2002)

42. EF Flushing, J Nagi, GA Di Caro, in International Conference on Computing, Networking and Communications (ICNC). A mobility-assisted protocol for supervised learning of link quality estimates in wireless networks (Hawaii, USA, Feb 2012), pp. 137-143. doi:10.1109/ICCNC.2012.6167397

43. MH Alizai, O Landsiedel, K Wehrle, A Becher, in Proceedings of the 7th GI/ITG Fachgespraech Wireless Sensor Networks. Challenges in short-term wireless link quality estimation, (2008)

44. A Becher, in Hot EmNetS'08: Proceedings of Fifth ACM Workshop on Embedded Networked Sensors. Towards short-term wireless link quality estimation (Virginia, USA, June 2008)

45. X Liu, M Haenggi, Throughput analysis of fading sensor networks with regular and random topologies. EURASIP J. Wireless Commun. 2005, 554-564 (2005)

46. R Bellman, On a routing problem. Q. Appl. Math. 16, 87-90 (1958)

47. EW Dijkstra, A note on two problems in connexion with graphs. Numerische Mathematik. 1(1), 269-271 (1959)

48. D Bertsekas, R Gallager, Data Networks, 2nd ed. (Prentice-Hall, Inc., Upper Saddle River, NJ, USA, 1992)

49. L Hu, D Evans, in Tenth Annual International Conference on Mobile Computing and Networking. Localization for mobile sensor networks (ACM Philadelphia, Pennsylvania, 2004)

50. M Lindhe, $\mathrm{KH}$ Johansson, Using robot mobility to exploit multipath fading. IEEE Wireless Commun. 16(1), 30-37 (2009)

51. V Loscri, E Natalizio, T Razafindralambo, N Mitton, in Proceedings of the 2013 IEEE International Conference on Distributed Computing in Sensor Systems. DCOSS '13. Distributed algorithm to improve coverage for mobile swarms of sensors (IEEE Computer Society, Washington, DC, 2013), pp. 292-294

52. J Kennedy, R Eberhart, in International Conference on Neural Networks. Particle swarm optimization, vol. 4 (IEEE, Perth WA, 1995), pp. 1942-1948

53. M Zubair, MAS Choudry, A Naveed, IM Qureshi, Particle swarm with soft decision for multiuser detection of synchronous multicarrier CDMA. IEICE Trans. Commun. E91-B(5), 1640-1643 (2008)

54. GF Franklin, DJ Powell, A Emami-Naeini, Feedback Control of Dynamic Systems, 4th edn. (Prentice Hall PTR, Upper Saddle River, NJ, USA, 2001)

55. J Kennedy, RC Eberhart, Swarm Intelligence. (Morgan Kaufmann Publishers Inc., San Francisco, CA, USA, 2001)

56. SIG SIG, Bluetooth, Specification of the Bluetooth system. Core Version. 1,2 (2003)

doi:10.1186/1687-1499-2014-207

Cite this article as: Fida et al:: Communication- and position-aware reconfigurable route optimization in large-scale mobile sensor networks. EURASIP Journal on Wireless Communications and Networking 2014 2014:207. 